\title{
Striatal Nurr1 Facilitates the Dyskinetic State and Exacerbates Levodopa-Induced Dyskinesia in a Rat Model of Parkinson's Disease
}

\author{
Rhyomi C. Sellnow, ${ }^{1,2}$ Kathy Steece-Collier, ${ }^{2}$ Feras Altwal, ${ }^{3}$ Ivette M. Sandoval, ${ }^{2,4}$ Jeffrey H. Kordower, ${ }^{5}$ \\ Timothy J. Collier, ${ }^{2}{ }^{\circledR}$ Caryl E. Sortwell, ${ }^{2}$ Anthony R. West, ${ }^{3}$ and ${ }^{\circledR}$ Fredric P. Manfredsson ${ }^{2,4}$ \\ ${ }^{1}$ Cell and Molecular Biology Program, Michigan State University, East Lansing, Michigan $48824,{ }^{2}$ Department of Translational Neuroscience, \\ Michigan State University, Grand Rapids, Michigan $49503,{ }^{3}$ Rosalind Franklin University, North Chicago, Illinois 60064, ${ }^{4}$ Department of \\ Neurobiology, Barrow Neurological Institute, Phoenix, Arizona 85013, and ${ }^{5}$ Rush University Medical Center, Chicago, Illinois 60612
}

The transcription factor Nurrl has been identified to be ectopically induced in the striatum of rodents expressing L-DOPAinduced dyskinesia (LID). In the present study, we sought to characterize Nurrl as a causative factor in LID expression. We used rAAV2/5 to overexpress Nurr1 or GFP in the parkinsonian striatum of LID-resistant Lewis or LID-prone Fischer-344 (F344) male rats. In a second cohort, rats received the Nurrl agonist amodiaquine $(\mathrm{AQ})$ together with L-DOPA or ropinirole. All rats received a chronic DA agonist and were evaluated for LID severity. Finally, we performed single-unit recordings and dendritic spine analyses on striatal medium spiny neurons (MSNs) in drug-naïve rAAV-injected male parkinsonian rats. rAAV-GFP injected LID-resistant hemi-parkinsonian Lewis rats displayed mild LID and no induction of striatal Nurrl despite receiving a high dose of L-DOPA. However, Lewis rats overexpressing Nurrl developed severe LID. Nurrl agonism with AQ exacerbated LID in F344 rats. We additionally determined that in L-DOPA-naïve rats striatal rAAV-Nurr1 overexpression (1) increased cortically-evoked firing in a subpopulation of identified striatonigral MSNs, and (2) altered spine density and thin-spine morphology on striatal MSNs; both phenomena mimicking changes seen in dyskinetic rats. Finally, we provide postmortem evidence of Nurr1 expression in striatal neurons of L-DOPA-treated PD patients. Our data demonstrate that ectopic induction of striatal Nurr1 is capable of inducing LID behavior and associated neuropathology, even in resistant subjects. These data support a direct role of Nurr1 in aberrant neuronal plasticity and LID induction, providing a potential novel target for therapeutic development.

Key words: dopamine; levodopa induced dyskinesia; nurr1; Parkinson's disease; plasticity

\section{Significance Statement}

The transcription factor Nurrl is ectopically induced in striatal neurons of rats exhibiting levodopa-induced dyskinesia [LID; a side-effect to dopamine replacement strategies in Parkinson's disease (PD)]. Here we asked whether Nurrl is causing LID. Indeed, rAAV-mediated expression of Nurrl in striatal neurons was sufficient to overcome LID-resistance, and Nurr1 agonism exacerbated LID severity in dyskinetic rats. Moreover, we found that expression of Nurr1 in L-DOPA naïve hemi-parkinsonian rats resulted in the formation of morphologic and electrophysiological signatures of maladaptive neuronal plasticity; a phenomenon associated with LID. Finally, we determined that ectopic Nurrl expression can be found in the putamen of L-DOPA-treated PD patients. These data suggest that striatal Nurr1 is an important mediator of the formation of LID.

Received Dec. 10, 2019; revised Mar. 13, 2020; accepted Mar. 19, 2020.

Author contributions: R.C.S., K.S.-C., A.R.W., and F.P.M. designed research; R.C.S., K.S.-C., F.A., I.M.S., T.J.C., C.E.S., A.R.W., and F.P.M. performed research; J.H.K. contributed unpublished reagents/analytic tools; R.C.S., K.S.-C., F.A., I.M.S., T.J.C., A.R.W., and F.P.M. analyzed data; R.C.S., K.S.-C., I.M.S., J.H.K., T.J.C., A.R.W., and F.P.M. wrote the paper.

This work was supported by the National Institute of Neurological Disorders and Stroke NS098079 (F.P.M., K.S.-C., A.R.W.) and a Target Advancement Program Grant from the Michael J. Fox Foundation (K.S.-C.). We thank J. Amiel Rosenkranz and Kuei Tseng for intellectual input and assistance with the design of electrophysiological studies; and Nathan Kuhn, Brian Daley, Jennifer Stancati, and Christopher Kelp for surgical and histological assistance.

K.S.C., F.P.M., T.J.C., and C.E.S. hold a patent describing genetic targeting of Nurr1 in the treatment of LID. The remaining authors declare no competing financial interests.

Correspondence should be addressed to Fredric P. Manfredsson at fredric.manfredsson@barrowneuro.org.

https://doi.org/10.1523/JNEUROSCI.2936-19.2020

Copyright $\odot 2020$ the authors

\section{Introduction}

Levodopa (L-DOPA) is considered the gold-standard pharmacotherapy for ameliorating motor symptoms in Parkinson's disease (PD). The precursor to dopamine (DA), L-DOPA alleviates motor symptoms by restoring striatal DAergic tone following the loss of DA from degenerated substantia nigra pars compacta (SNc) neurons (Cotzias et al., 1967; Fox et al., 2011). Unfortunately, chronic L-DOPA treatment leads to the development of druginduced abnormal involuntary movements (AIMs) in most PD patients (Ahlskog and Muenter, 2001; Manson et al., 2012) known as L-DOPA-induced dyskinesia (LID). These are disruptive hyperkinetic and dystonic movements associated with 
supra-physiological plasma L-DOPA levels. However, the underlying cause of LID remains largely unknown.

A previous study looking at gene expression differences between the direct and indirect striatal output pathways of the basal ganglia in dyskinetic mice showed a marked increase in expression of the transcription factor Nurr1 in direct pathway medium spiny neurons (MSNs; Heiman et al., 2014). The induction of Nurr1 expression in striatal MSNs of dyskinetic animals is particularly noteworthy because Nurr1 is not normally expressed in striatal neurons (Xiao et al., 1996). Similarly, we and others have observed that Nurr1 mRNA colocalizes with markers of both the direct and indirect pathway in the rat dyskinetic striatum following dyskinesiogenic L-DOPA dosing, with a higher abundance in the direct pathway (Sodersten et al., 2014; Sellnow et al., 2015). These findings provide compelling evidence that LDOPA in the DA depleted striatum appears to induce ectopic Nurr1 expression, however, the importance/role of Nurr1 in LID formation remains unknown.

Nurr1 is an orphan nuclear transcription factor and a member of the NR4A family (Giguere, 1999) and its expression is crucial for DAergic neuronal development and long-term function. Indeed, Nurr1 knock-out mice are not viable, and Nurr1+/heterozygotes show DA dysfunction and loss of SNc DA neurons (Zetterstrom et al., 1997; Jiang et al., 2005; Kadkhodaei et al., 2009). Nurr1 expression in the SNc decreases with age in humans, and in fact, multiple Nurr1 isoforms are associated with familial forms of PD (Dekker et al., 2003; Le et al., 2003). Accordingly, Nurr1-based therapeutics are of great interest as potential disease-modifying treatments for PD. However, although Nurr1 in the SN may be of benefit in PD, its activity in the striatum warrants further examination (Kim et al., 2015; Smith et al., 2015; Dong et al., 2016).

Much research has focused on LID-associated changes in the physiology of the basal ganglia and its connecting target nuclei. In vivo recordings of $\mathrm{PD}$ patients with deep brain stimulation therapy have revealed impaired depotentiation in basal ganglia output centers associated with severe LID (Prescott et al., 2014). Similar findings in preclinical studies demonstrate a loss of bidirectional plasticity associated with LID (Picconi et al., 2003). Specifically, following DAergic denervation, the balance between long-term potentiation (LTP) and long-term depression (LTD) is lost in the striatum, indicating pathologic function and abnormal neuroplasticity in corticostriatal transmission. Although LDOPA treatment restores this corticostriatal plasticity, LTD is not present when LID develops (Picconi et al., 2003) and an imbalance or dysfunction of striatal output is thought to be a key factor in LID expression (Ryan et al., 2018).

Some of these LID-associated changes in striatal plasticity are thought to be related to changes in dendritic spine morphology. Indeed, striatal spine density and morphology are dramatically altered in animal models following LID induction (Zhang et al., 2013; Fieblinger et al., 2014; Nishijima et al., 2014; Suarez et al., 2014; Maiti et al., 2015). These structural elements are dynamic, and their proliferation or pruning can occur because of normal neuronal processes such as motor learning or can be indicative of pathologic states (Bagetta et al., 2010; Spiga et al., 2014; Maiti et al., 2015). As in PD patients (McNeill et al., 1988), animal models of PD have revealed dramatic spine loss following DA depletion, and in preclinical models, reestablishment of spine density occurs with L-DOPA (Zhang et al., 2013; Fieblinger et al., 2014; Nishijima et al., 2014; Suarez et al., 2014, 2016; Villalba and Smith, 2018). However, whereas L-DOPA administration results in normalization of total spine density in some MSNs
(Fieblinger et al., 2014; Suarez et al., 2014), LID is associated with maladaptive spine changes including a significant increase in the number of large mushroom spines, multisynaptic spines, and alterations in corticostriatal synapses (Zhang et al., 2013; Fieblinger et al., 2014; Suarez et al., 2014). Thus, changes in spine density, morphology, and corticostriatal transmission are important pathophysiological factors associated with LID expression. However, our understanding of underlying triggers or causes of these aberrant modifications remains limited.

We posit that Nurr1 is a critical trigger and master regulator necessary for these maladaptive plasticity changes and the induction/expression of LID. Indeed, Nurr1 expression is induced in several cell types by multiple stimuli including stress, addiction, and learning and memory. Within the learning and memory circuits of the hippocampus, Nurr1 is induced in conjunction with associative learning and is required for long-term memory formation (Pena de Ortiz et al., 2000; Colon-Cesario et al., 2006; Hawk et al., 2012). Nurr1 also plays a key role in the remodeling of basal ganglia circuits during addiction (Campos-Melo et al., 2013). It is thus reasonable to suggest that Nurr1 may play a similar role in the aberrant plasticity associated with dyskinesiogenesis; a form of maladaptive motor learning. To determine whether Nurr1 is a key contributor to LID development we examined whether it's overexpression, in the absence or presence of LDOPA, could induce dyskinesia expression and/or the structural and physiological correlates of LID in striatal MSN. We used gene therapy and pharmacological manipulations to modulate expression and activity of Nurr1 in MSNs of hemi-parkinsonian rats and evaluated the effect on dyskinetic behaviors and MSN structure and function.

\section{Methods}

Adeno-associated virus production

Vector design and production methods were used as described previously (Benskey et al., 2016). Briefly, the human Nurr1 or the GFP coding sequence were cloned into an rAAV genome under control of the CAG promoter. The genome was packaged into rAAV2/5 via double transfection of HEK293 cells with rAAV genome and the helper plasmid pXYZ5 as previously described (Sandoval et al., 2019). Virus was purified using an iodixanol gradient and concentrated in concentration columns. Viral titer was ascertained by dot blot and adjusted to a working titer of $1.0 \times 1013 \mathrm{vg} / \mathrm{ml}$.

\section{Animals and surgeries}

Adult male Sprague-Dawley (SD), Lewis, and Fischer-344 (F344) rats (200-220 g on arrival; Charles River Laboratories) were used in the studies. Studies were conducted in accordance with Institutional Animal Care and Use Committee approval of Michigan State University (AUF MSU06/16-093-00) and Rosalind Franklin University (AUF A3279-01). Our investigation into Nurr1 as a factor in LID was based on work examining intrinsic genetic differences in the SD strain and the relation of these differences to LID. Thus, initial experimentation was performed on SD rats. However, a portion of SD rats are LID resistant (Konradi et al., 2004; Zhang et al., 2013), adding significant attrition to the experimentation. We therefore switched to Fischer rats, which all develop LID (Sellnow et al., 2019). The inclusion of Lewis rats was based on our preceding work (SteeceCollier et al., 2020) where we demonstrate (1) the resistance of this strain to LID, and (2) the result of an in-depth transcriptional analysis comparing the two strains. 
Rats were housed two per cage up until LID behavior testing began, when they were separated and singly housed in enriched environment conditions. Animals were housed in a light-controlled (12 h light/dark cycle) and temperature-controlled $\left(22 \pm 1^{\circ} \mathrm{C}\right)$ room and had ad libitum access to standard laboratory chow and water.

All stereotaxic surgeries were performed under $2 \%$ isoflurane. After being anesthetized, animals were placed in a stereotaxic frame and 6-hydroxydopamine (6-OHDA) hydrobromide or rAAV was delivered via a glass capillary needle fitted to a Hamilton syringe (Benskey and Manfredsson, 2016). Three weeks following lesion surgery, animals were tested for spontaneous forepaw use (cylinder test) to estimate lesion efficacy (Sellnow et al., 2019). Rats were matched into vector groups based on cylinder test forepaw deficits to ensure equal lesions between the treatment groups. Animals in DA and Nurr1 agonist studies did not receive vector.

Lesions were performed using $5 \mathrm{mg} / \mathrm{ml} 6$-OHDA mixed in $0.2 \mathrm{mg} / \mathrm{ml}$ ascorbic acid immediately preceding injections. SD rats used for spine analysis were lesioned with two $2 \mu$ injections of 6-OHDA (10 $\mu \mathrm{g}$ per injection) in the left striatum [first injection from bregma: Anterior posterior (AP): $+1.6 \mathrm{~mm}$, medial lateral (ML): $2.4 \mathrm{~mm}$, dorsal ventral (DV): $-4.2 \mathrm{~mm}$ from skull; second injection from first injection site: AP: $-1.4 \mathrm{~mm}$, ML: $+0.2 \mathrm{~mm}$, DV: $-2.8 \mathrm{~mm}$ ]. F344 and Lewis rats used in AIM behavior and in vivo cell recordings received two $2 \mu \mathrm{l}$ injections of 6-OHDA $(10 \mu \mathrm{g}$ per injection), one in the medial forebrain bundle (from bregma: AP: $-4.3 \mathrm{~mm}$, ML: $+1.6 \mathrm{~mm}$, DV: -8.4 $\mathrm{mm}$ from skull) and one in the SNc (from bregma: AP: -4.8 $\mathrm{mm}, \mathrm{ML}:+1.7 \mathrm{~mm}, \mathrm{DV}:-8.0 \mathrm{~mm}$ from skull). The needle was lowered to the site and the injection began after $30 \mathrm{~s}$. The needle was removed 2 min after the injection was finished and cleaned between each injection. Lesion efficacy was estimated 2.5 weeks following 6-OHDA injection with the cylinder task as previously described (Sellnow et al., 2019).

Viral delivery surgeries were performed similarly 3 weeks following 6-OHDA lesions, as described previously (Benskey and Manfredsson, 2016). Animals in the LID behavior overexpression studies received a single $2 \mu$ injection of either rAAV-Nurr 1 or rAAV-GFP targeting lateral striatum (from bregma: AP: + 0.5 $\mathrm{mm}, \mathrm{ML}:+3.7 \mathrm{~mm}, \mathrm{DV}:-5.3 \mathrm{~mm}$ from skull). Animals used in morphologic and electrophysiology studies received two $2 \mu \mathrm{l}$ injections of either rAAV-Nurr1 or rAAV-GFP targeting the entire striatum to ensure that all sampled neurons were transduced (first injection from bregma: AP: $+1.0 \mathrm{~mm}$, ML: +3.0 $\mathrm{mm}, \mathrm{DV}:-4.0 \mathrm{~mm}$ from dura; second injection from bregma: AP: $-1.6 \mathrm{~mm}, \mathrm{ML}:+3.8 \mathrm{~mm}, \mathrm{DV}:-5 \mathrm{~mm}$ from dura).

\section{L-DOPA therapy and abnormal involuntary movements ratings}

Animals in electrophysiology and spine analysis experiments were not treated with L-DOPA or DA agonists before recordings or sacrifice, and therefore remained non-dyskinetic. For behavioral studies, drug-induced dyskinesia severity was evaluated using the AIM scale (Lundblad et al., 2002; Steece-Collier et al., 2003) by an observer blinded to treatment conditions. F344 and Lewis rats received subcutaneous injections of either increasing doses of L-DOPA (2-8 $\mathrm{mg} / \mathrm{kg})$ with benserazide $(12 \mathrm{mg} / \mathrm{kg})$, or the DA receptor agonists SKF-81 $297(0.8 \mathrm{mg} / \mathrm{kg})$ and quinpirole $(0.2 \mathrm{mg} / \mathrm{kg})$. Dosing occurred $3 \mathrm{~d} /$ week for 3 weeks for L-DOPA studies and 1 week for DA agonist studies. The AIM scale was used to rate drug-induced AIMs, as previously described (SteeceCollier et al., 2003; Maries et al., 2006). Briefly, AIM severity is evaluated by a blinded observer scoring the level of dystonia of the limbs and body, hyperkinesia of the forelimbs, and hyperoral movements. Each AIM is given two scores; one indicating the intensity $(0=$ absent, $1=$ mild, $2=$ moderate, $3=$ severe $)$ and frequency $(0=$ absent, $1=$ intermittently present for $<50 \%$ of the observation period, $2=$ intermittently present for $>50 \%$ of the observation period, $3=$ uninterruptable and present through the entire rating period). Each AIM is given a severity score by multiplying the intensity and frequency, and the overall AIM score for each time point is a sum of severity for all behaviors. The sum of all AIM scores from each time point makes up the total AIM score. Peak-dose dyskinesia is considered to be $75 \mathrm{~min}$ post-drug administration. An animal is considered non-dyskinetic with a score of $\leq 4$ ((Maries et al., 2006). Animals were observed in $25 \mathrm{~min}$ increments following drug delivery until AIMs subsided.

\section{Pharmacological activation of Nurr1 with L-DOPA administration} To assess the impact of pharmacological activation of Nurr1 on LID expression, we used the Nurr1 agonist AQ, which activates the transcriptional function of Nurr1 through physical interaction with its ligand binding domain and provides neuroprotection against 6-OHDA-induced SN DA neuron loss in an in vivo rat model (Kim et al., 2015). In all studies employing AQ we used the previously reported neuroprotective dose $(20 \mathrm{mg} / \mathrm{kg})$. Pretreatment with AQ (20 mg/kg twice per day; Kim et al., 2015) was initiated before the introduction of L-DOPA. The rationale for pretreatment was that potential neuroprotective therapy for PD involving Nurrl agonists would presumably be initiated soon after diagnosis and before introduction of L-DOPA, which typically occurs $\sim 1$ year after diagnosis (Simuni, 2018). To determine whether AQ pretreatment exacerbated LID induction, adult male $\mathrm{SD}$ rats rendered unilaterally parkinsonian with 6-OHDA (as described in Animals and Surgeries) first received a low dose $(3 \mathrm{mg} / \mathrm{kg}$ ) of L-DOPA for 1 week (M-Fr). For all doses, levodopa was administered with $12 \mathrm{mg} / \mathrm{kg}$ benserazide and $60 \mathrm{~min}$ after AQ or the vehicle saline. The dose of L-DOPAwas next increased to a moderate dose $(6 \mathrm{mg} / \mathrm{kg})$, which was given daily for 2 weeks in the presence of AQ or saline. The dose was finally escalated to a high dose $(12 \mathrm{mg} / \mathrm{kg}$ ) for an additional week to determine whether LID severity could be further escalated with high-dose L-DOPA in the presence of AQ. LID were rated at 75 min post-LDOPA("peak dose") and at time points indicated in Figure $6 \mathrm{~A}$.

\section{Pharmacological activation of Nurr1 with ropinirole administration}

Ropinirole is a non-ergoline D2/D3 DA agonist used for the treatment of motor dysfunction in early PD, and as adjunct therapy along with L-DOPA in advanced stages of the disease (Zesiewicz et al., 2017a,b). In early PD, monotherapy with ropinirole has significantly less dyskinesia liability than levodopa in PD patients (Hauser et al., 2007; Bastide et al., 2015; Zesiewicz et al., 2017a,b) and parkinsonian rodents (Lundblad et al., 2002; Carta et al., 2008; Lane and Dunnett, 2010). Additional rationale for examining the interaction of ropinirole with Nurrl agonist neuroprotective therapy is that neuroprotective drugs would most likely be administered to individuals with PD early in the disease, a time when monotherapy with DA agonists like ropinirole is most common.

Before undertaking the reported studies, a pilot study was done to establish a dose of ropinirole that in our hands would produce stable, minimal dyskinetic behavior (e.g., total dyskinesia severity score of $<10$ in the absence of adjunct therapy over 2 weeks). The necessity for this pilot dose-response study was based on the fact that there is a significant range of doses of 
ropinirole reported in the literature to induce dyskinesias and/or rotational behavior ranging from $0.04 \mathrm{mg} / \mathrm{kg}$ (Millan et al., 2004) to $20 \mathrm{mg} / \mathrm{kg}$ (Papathanou et al., 2011), as well as doses in between. We found $0.2 \mathrm{mg} / \mathrm{kg}$ to fulfil the desired criterion. This dose was increased to $0.5 \mathrm{mg} / \mathrm{kg}$ for a second time frame of $9 \mathrm{~d}$ as depicted in Figure 6.

To determine whether AQ pretreatment exacerbated dyskinesia induction following chronic ropinirole administration, parkinsonian rats received once-daily $\mathrm{AQ}$ or the vehicle saline for 1 week beginning 3-4 weeks after 6-OHDA. To determine whether AQ pretreatment exacerbated ropinirole-induced dyskinesias (RID) induction, unilaterally parkinsonian rats first received a low dose $(0.2 \mathrm{mg} / \mathrm{kg})$ of ropinirole for 3 weeks (MFr). The dose was escalated to $0.5 \mathrm{mg} / \mathrm{kg}$ for an additional 2 weeks. Peak RID occurred at $20 \mathrm{~min}$ post-injection, and was rated at time points indicated in Figure $6 D$.

In vivo single-unit recordings

Animals used for electrophysiology were shipped to Rosalind Franklin University 3 weeks following vector delivery and acclimatized for at least 3 weeks before initiation of recordings. F344 rats were deeply anesthetized with urethane $(1.5 \mathrm{~g} / \mathrm{kg}$ in physiological saline). In vivo extracellular single-cell recordings of electrophysiologically identified striatal MSNs (Threlfell et al., 2009; Sammut et al., 2010; Padovan-Neto et al., 2015), and in some cases striatonigral projection neurons were measured in vector-treated F344 rats without L-DOPA treatment, or in established dyskinetic animals. Electrical stimulation and antidromic activation of striatonigral neurons was performed as previously described (Threlfell et al., 2009; Sammut et al., 2010). For antidromic stimulation, an electrode was placed in the ipsilateral substantia nigra pars reticulata (SNr; from bregma: AP: -5.0 $\mathrm{mm}$, ML: $+2.5 \mathrm{~mm}$, DV: $-8.0 \mathrm{~mm}$ from dura). For striatal single-unit recording ipsilateral to cortical and $\mathrm{SNr}$ stimulation, extracellular microelectrodes were lowered slowly through the dorsolateral striatum (from bregma: AP: $+0-0.75$ $\mathrm{mm}$, ML: $+3.3-3.7 \mathrm{~mm}$, DV: -3.0 to $6.5 \mathrm{~mm}$ from dura) while electrical stimuli were delivered to the cortex to isolate responsive striatal single-units. The order of stimulation trials was counterbalanced across stimulus intensities/stimulation trials (i.e., either $400-1000$ or $1000-4000 \mu \mathrm{A}$ ). Spikes evoked by stimulating the $\mathrm{SNr}$ were determined to be antidromically activated based on numerous criteria including spike collision with orthodromic spikes, fixed action potential latency, and identification of the spike threshold for all or none spiking occurring consistently over 10 trials (Threlfell et al., 2009; Sammut et al., 2010; Padovan-Neto et al., 2015).

\section{Tissue collection and immunohistochemistry}

Animals received a final injection of either L-DOPA or DA agonists $2 \mathrm{~h}$ before euthanasia. Rats were anesthetized deeply with a lethal dose of sodium pentobarbital, and intracardially perfused with Tyrode's solution (in mM: 137 sodium chloride, 1.8 calcium chloride dihydrate, 0.32 sodium phosphate monobasic dihydrate, 5.5 glucose, 11.9 sodium bicarbonate, 2.7 potassium chloride) followed by $4 \%$ paraformaldehyde (PFA). Brains were rapidly removed and postfixed for $72 \mathrm{~h}$ in $4 \%$ PFA before being transferred into $30 \%$ sucrose. Brains were sectioned on a freezing sliding microtome at $40 \mu \mathrm{m}$ and stored at $-20^{\circ} \mathrm{C}$ in cryoprotectant (30\% ethylene glycol, $0.8 \mathrm{~mm}$ sucrose in $0.5 \times$ tris-buffered saline).

Immunohistochemistry (IHC) was performed as previously reported (Benskey et al., 2018). A 1:6 series of free-floating tissue was stained for TH (MAB318, MilliporeSigma) Nurr1 (AF2156, R\&D Systems) or GFP (AB290, Abcam). Briefly, sections were washed in $1 \times$ TBS with $0.25 \%$ Triton X-100, incubated in $0.3 \%$ $\mathrm{H}_{2} \mathrm{O}_{2}$ for $30 \mathrm{~min}$, and rinsed and blocked in $10 \%$ normal goat or donkey serum for $2 \mathrm{~h}$. Tissue was incubated in primary antibody (TH 1:4000, Nurr1 $1.5 \mu \mathrm{g} / \mathrm{ml}$, GFP 1:20,000) overnight at room temperature. After washing, tissue was incubated in secondary antibody (Biotinylated horse anti-mouse IgG 1:500, BA-2001, Vector Laboratories; biotinylated donkey anti-goat IgG 1:500, AP180B, Millipore-Sigma; biotinylated goat anti-rabbit IgG 1:500, AP132B, Millipore-Sigma) followed by the Vectastain ABC kit (Vector Laboratories). Tissue staining was developed

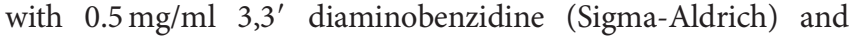
$0.03 \% \mathrm{H}_{2} \mathrm{O}_{2}$. Sections were mounted on slides, dehydrated, and coverslipped with Cytoseal (ThermoFisher).

\section{IHC for Nurr1 in human striatal tissue}

We examined striatal brain sections from older adults diagnosed with PD with dyskinesia or dementia with Lewy bodies (DLB; sections from 3 subjects were screened) as control. Patient diagnosis was performed by movement disorders specialists at the Rush University Movement Disorders Clinic. Subjects: average age: 78.3, average UPDRS III (on): 50, all subjects were Hoehn\& Yahr stage 4 at time of death. All subjects signed an informed consent for clinical evaluation. Postmortem consent was provided by next of kin or a legal representative. The Human Investigation Committee at Rush University Medical Center approved this study.

Free-floating striatal sections were washed in $1 \times$ TBS with $0.4 \%$ Triton six times for $10 \mathrm{~min}$. Sections were incubated $0.3 \%$ $\mathrm{H}_{2} \mathrm{O}_{2}$ for $45 \mathrm{~min}$, rinsed, mounted on slides, and allowed to dry overnight. Antigen retrieval was performed using Antigen Unmasking Solution (Vector Laboratories) at $80^{\circ} \mathrm{C}$ for $30 \mathrm{~min}$, and then removed from heat and allowed to cool in solution for $30 \mathrm{~min}$. Following washing, slides were incubated in 10\% NDS serum for $4 \mathrm{~h}$. Slides were incubated in Nurr1 antibody $(1.5 \mu \mathrm{g} /$ $\mathrm{ml}$ ) overnight. Slides were then washed, incubated in secondary antibody (donkey anti goat 1:500) for $4 \mathrm{~h}$, followed by incubation in $A B C$ for $1 \mathrm{~h}$. Nurr1 protein was visualized using Vector SG Peroxidase Substrate Kit (Vector Laboratories). Slides were then coverslipped with Cytoseal (ThermoFisher).

\section{In situ hybridization}

In situ hybridization with IHC was performed using the RNAscope 2.5 HD Duplex Assay according to the manufacturer's protocol (Advanced Cell Diagnostics). Forty micrometer striatal sections were treated with the hydrogen peroxide solution for at least $10 \mathrm{~min}$, or until active bubbling from the tissue subsided. Sections were washed in $1 \times$ TBS, mounted onto slides, and allowed to dry for at least $48 \mathrm{~h}$. Slides were then boiled for $10 \mathrm{~min}$ in the Target Retrieval solution, followed by Protease Plus treatment. Tissue was hybridized with target probe for direct and indirect pathway markers [dopamine receptor 1 (D1) or enkephalin (Enk), respectively; Lu et al., 1997] for $2 \mathrm{~h}$ at $40^{\circ} \mathrm{C}$. Slides were rinsed in $1 \times$ RNAscope Wash Buffer. Sequential amplification steps were then applied to the slides, with $1 \times$ wash buffer rinses between each amplification. After the sixth amplification, the probe was visualized using the Detect Red Signal solution for $10 \mathrm{~min}$.

Immediately following in situ hybridization, the tissue was stained immunohistochemically for Nurr1. IHC was performed as described, and Nurr1 protein was visualized using Vector SG Peroxidase kit (Vector Laboratories). 
A

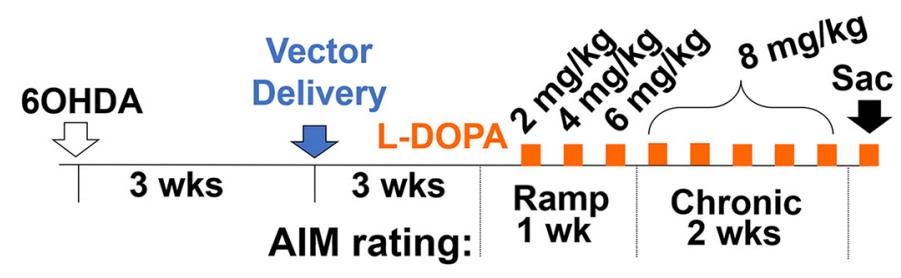

B TH D
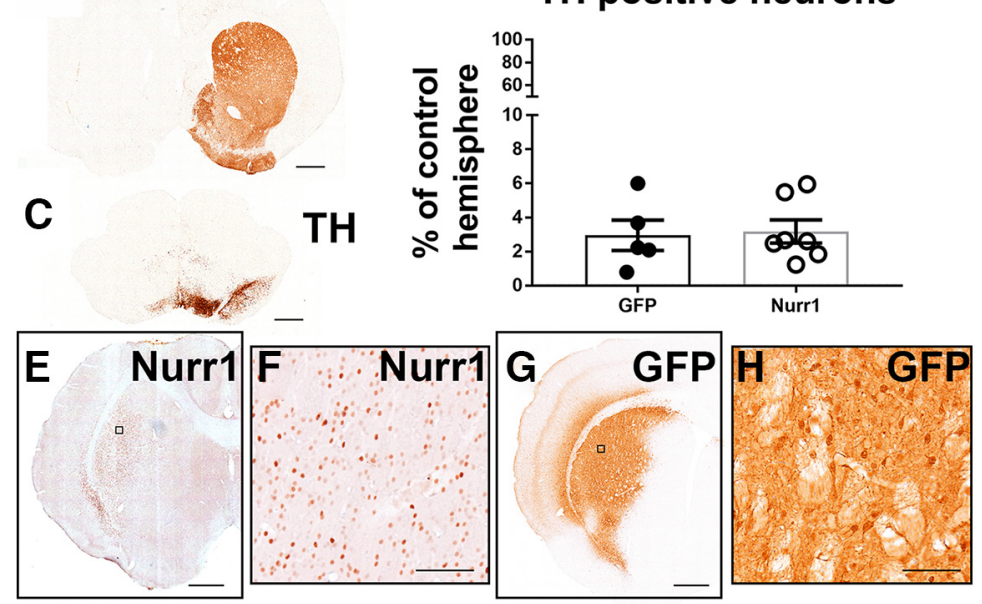

Figure 1. Experimental design and model validation. $A$, Experimental timeline showing timing of surgeries and LID induction. AIM scores were evaluated as indicated by squares. $\boldsymbol{B}-\boldsymbol{D}, 6-0 \mathrm{HDA}$ injections caused near-complete loss of TH fibers in the striatum (B) as well as TH-positive cells in the SNc (C). Stereology confirmed that all animals had $>94 \%$ TH cell loss in the SNc (D). $\boldsymbol{E}-\boldsymbol{H}$, Nurr1 $(\boldsymbol{E}, \boldsymbol{F})$ and GFP $(\boldsymbol{G}, \boldsymbol{H})$ vector-mediated expression were confirmed in the striatum with IHC. Scale bars: $\boldsymbol{B}, \boldsymbol{C}, \boldsymbol{E}$, $H, 1 \mathrm{~mm} ; \boldsymbol{F}, \boldsymbol{H}, 100 \mu \mathrm{m}$.

\section{Golgi-Cox impregnation and spine analysis}

$\mathrm{SD}$ rats for spine analysis were perfused with Tyrode's solution followed by $4 \%$ PFA. Brains were removed and hemisected. The caudal portion of the brain was then postfixed in $4 \%$ PFA and used for lesion evaluation. The rostral portion was postfixed for $1 \mathrm{~h}$ and then transferred to $0.2 \mathrm{M}$ phosphate buffer until further processing. The rostral section was sectioned on a vibratome at $100 \mu \mathrm{m}$. Sections were then processed for Golgi-Cox impregnation as described previously (Levine et al., 2013). Briefly, sections were sandwiched gently between two glass slides and placed into the Golgi-Cox solution $(1 \%$ mercury chloride, $1 \%$ potassium chromate, $1 \%$ potassium dichromate) in the dark for $14 \mathrm{~d}$. Sections were transferred into a $1 \%$ potassium dichromate solution for $24 \mathrm{~h}$. Sections were mounted on $4 \%$ gelatin-coated slides and the stain was developed with $28 \%$ ammonium hydroxide followed by $15 \%$ Kodak fixer. Slides were dehydrated in alcohol and xylene and coverslipped.

Spine density and morphology were quantified using Neurolucida (MicroBrightField Bioscience) as previously described (Zhang et al., 2013). Neurons of the dorsal striatum were selected for analysis. To be selected for quantification, a neuron needed at least four primary dendrites that projected radially, not bidirectionally, from the cell body. One dendrite per neuron was traced and the spines quantified and typified. Each spine was typified into one of four classes: thin, mushroom, stubby, and branched (Maiti et al., 2015). Ten individual dendrites per hemisphere per animal were quantified. Spine quantity and phenotype were evaluated based on total dendrite length, or proximal (dendrite branch orders 1-2) or distal (branch orders 3 n) dendritic regions.

\section{Stereology}

Unbiased stereology was used to determine lesion status via TH loss as previously described (Benskey et al., 2018). Using Stereo Investigator software with the optical fractionator probe (MicroBrightField Bioscience), TH-positive neurons in every sixth section of the whole $\mathrm{SNc}$ were counted on the intact and lesioned hemisphere, giving an estimate of total TH-positive cells in the SNc. The coefficient of error for each estimate was calculated and was $<0.1$ (Gundersen, $m=1$ ).

\section{Statistical analysis}

Statistical analysis was performed using StatView v5.0 or GraphPad Prism v7.0 (GraphPad Software). All graphs were created in GraphPad. Lesion status was evaluated using unpaired, one-tailed $t$ tests. Mean AIMs were evaluated using a Mann-Whitney $U$ test or the KruskalWallis test. Differences between vector groups were compared with $p \leq 0.05$ being considered statistically significant. For Nurrl agonist studies, the means of the total LID or RID from each rating day were compared between treatment groups (LD+AQ vs $\mathrm{LD}+\mathrm{Veh}$ ) using Mann-Whitney $U$. Although the mean is the most common statistic used, to be most judicious based on the current international interest in Nurr1 activating drugs for patients with $\mathrm{PD}$, we also present here the median scores across time because extreme/outlier values do not affect the median as strongly as they do the mean. Median scores were grouped per dose and compared between LD+AQ and LD+Veh using an unpaired $t$ test. Differences in spine quantity and morphology were evaluated using unpaired $t$ tests. Differences across MSN recording sites within separate vector groups at each recording site and outcomes from animals were compared across experimental groups using a two-way repeated-measures ANOVA (vs Nurr1) $\times 2$ (vs drug treatment) with an $\alpha$ set to 0.05 . For all electrophysiological experiments, rat group sizes, and all $n$ values of MSNs recorded across vector groups were determined to be adequately powered for based on outcomes from power analyses performed. The potential two-way interaction between treatment groups was also examined to determine how treatment effects differ as a function of drug treatment or gene therapy (Padovan-Neto et al., 2015).

\section{Results}

rAAV transduction of Nurr1 does not exacerbate AIMs in LIDsusceptible but does in LID-resistant rats

We first aimed to determine whether ectopic Nurr1 expression can exacerbate LID in LID susceptible and resistant rats. All Fischer-344 (F344) and all Lewis rats included in the final analysis were lesioned with $\geq 94 \%$ TH loss (Fig. $1 B-D$ ), validated postmortem with stereological quantification of $\mathrm{TH}$ 
immunoreactivity in the SNc. Vector transduction and expression was confirmed with postmortem IHC in either rAAV-Nurr1 (F344: $n=7$, Lewis $n=5$ ) or rAAV- GFP (F344: $n=7$, Lewis $n=5)$ transduced rats (Fig. $1 E-H$ ). Vector-mediated expression results in robust Nurr1 expression in both F344 and Lewis rats. Notably, vector transduction resulted in markedly higher Nurr1 expression than what was seen in rAAV-naïve, LID + F344 animals, where Nurr1 is ectopically upregulated in response to dyskinesiogenic $\mathrm{L}^{-}$ DOPA (Fig. 2A,C). rAAV-naïve Lewis animals, however, express much lower level AIMs than rAAV-naïve F344 rats, and also do not ectopically express Nurr1 in the striatum (Fig. 2B).

AIMs testing began 3 weeks following virus delivery to ensure maximal transgene expression before animals being placed on a L-DOPA treatment paradigm (for experimental timeline, see Fig. $1 A$ ). To evaluate AIMS, animals were first challenged with vehicle $(0 \mathrm{mg} /$ $\mathrm{kg}$ L-DOPA, $12 \mathrm{mg} / \mathrm{kg}$ benserazide) to determine whether Nurr1 overexpression would cause drug-independent AIMs. No vector group showed AIMs following vehicle administration (Fig. 3). When treated with L-DOPA, F344 treated with rAAV-Nurr1 or GFP rats showed no significant differences in total AIM scores at any rating time point [Fig. 5A-D; total AIM sum: D1 $2 \mathrm{mg} / \mathrm{kg}$ rAAV-Nurr1 (Median $[\mathrm{Md}]=0.5)$ rAAV-GFP $(\mathrm{Md}=1), U=17, p>0.05$; D3 $4 \mathrm{mg} / \mathrm{kg}$ rAAV-Nurr1 (Md=8) rAAV-GFP $(\mathrm{Md}=6.5), U=10$, $p>0.05 ;$ D5 $6 \mathrm{mg} / \mathrm{kg}$ rAAV-Nurr1 $(\mathrm{Md}=39.5)$ rAAV-GFP $(\mathrm{Md}=29), U=14, p>0.05 ; \mathrm{D} 88 \mathrm{mg} / \mathrm{kg}$ rAAV-Nurrl $(\mathrm{Md}=55)$ rAAV-GFP $(\mathrm{Md}=42), U=12, p>0.05 ; \mathrm{D} 108 \mathrm{mg} / \mathrm{kg} \mathrm{rAAV}-$ Nurr1 (Md=61.5) rAAV-GFP $(\mathrm{Md}=52.5), \quad U=11, p>0.05$; D12 $8 \mathrm{mg} / \mathrm{kg}$ rAAV-Nurr1 $(\mathrm{Md}=57.5)$ rAAV-GFP $(\mathrm{Md}=48)$, $U=14, p>0.05$; D15 $8 \mathrm{mg} / \mathrm{kg}$ rAAV-Nurrl (Md=55) rAAVGFP (Md=63), $U=15, p>0.05 ;$ D $178 \mathrm{mg} / \mathrm{kg}$ rAAV-Nurr1 $(\mathrm{Md}=62.5) \mathrm{rAAV}-\mathrm{GFP}(\mathrm{Md}=54), U=13, p>0.05]$. Additionally, Nurr1 overexpression did not differentially impact individual attributes of LID (Fig. 4). These data suggest that additional induction of striatal Nurr1 above an apparent threshold does not exacerbate AIMs of LID-prone F344 rats.

In contrast, the normally LID-resistant Lewis rat treated with rAAV-Nurr1 developed severe LID when treated chronically with L-DOPA, whereas rAAV-GFP control rats expressed lowlevel AIMs (Fig. $5 E-H$ ). This was first observed at the $6 \mathrm{mg} / \mathrm{kg}$ treatment, in both total AIM score sum and peak-dose LID [total AIM sum $6 \mathrm{mg} / \mathrm{kg}$ rAAV-Nurr1 $(\mathrm{Md}=34)$ rAAV-GFP $(\mathrm{Md}=$ 3), $U=3, p<0.05$; peak-dose AIM $6 \mathrm{mg} / \mathrm{kg}$ rAAV-Nurr1 $(\mathrm{Md}=13.5)$ rAAV-GFP $(\mathrm{Md}=4), U=0, p<0.01]$. rAAV-Nurr 1treated Lewis rats also displayed more severe AIMs than their rAAV-GFP counterparts on Days 8,10 , and 19 with $8 \mathrm{mg} / \mathrm{kg} \mathrm{L-}$ DOPA [peak dose AIMs: Day 8 rAAV-Nurr1 $(\mathrm{Md}=16) \mathrm{rAAV}$ GFP $(\mathrm{Md}=3), U=2, p<0.05$; Day 10 rAAV-Nurr1 $(\mathrm{Md}=16)$ rAAV-GFP $(\mathrm{Md}=6), \quad U=0, p<0.01$; Day 17 rAAV-Nurr1

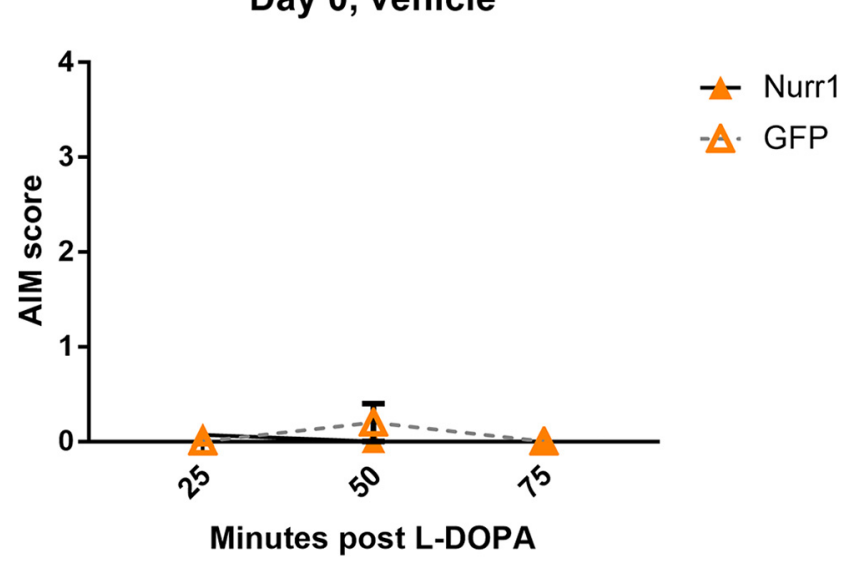

Figure 3. Striatal Nurr1 overexpression does not induce drug-independent AIMs. F344 rats treated with rAAV-Nurr1 or rAAV-GFP do not express AIMs when treated with vehicle $(0 \mathrm{mg} / \mathrm{kg}$ L-DOPA, $12 \mathrm{mg} / \mathrm{kg}$ benserazide) showing that Nurr1 overexpression does not promote AIMs without drug treatment.

$(\mathrm{Md}=13.5) \mathrm{rAAV}-\mathrm{GFP}(\mathrm{Md}=4), U=0, p<0.01$; Day $19 \mathrm{rAAV}-$ Nurr1 $(\mathrm{Md}=16) \quad$ rAAV-GFP $(\mathrm{Md}=3), \quad U=2, \quad p<0.05]$. Additionally, rAAV-Nurr1 Lewis AIM scores were indistinguishable from both rAAV-GFP and rAAV-Nurr1 F344 rats (Fig. $5 I-J)$. Together, these data shows that the expression of Nurr1 is sufficient to overcome resistance to severe LID seen in Lewis animals. 
A

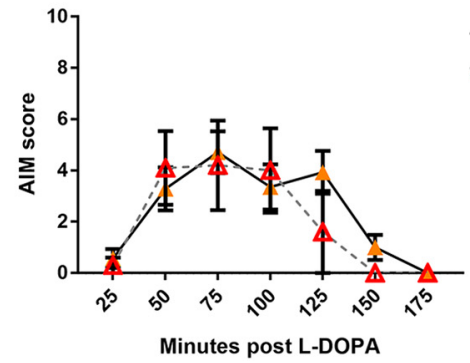

B

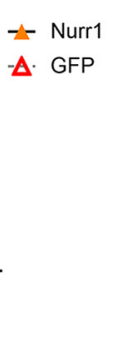

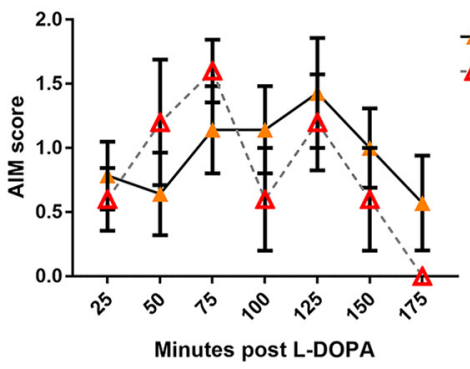

C

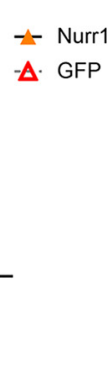

Forelimb AIMs

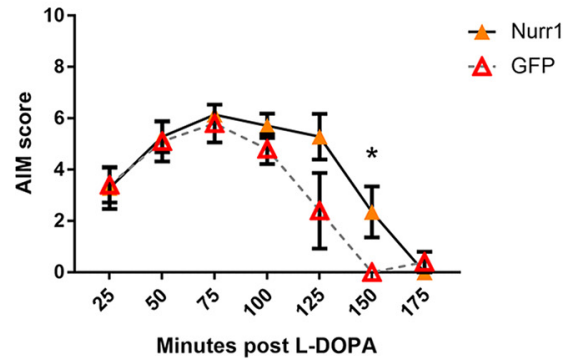

Figure 4. Nurr1 does not impact individual AOLs in LID-susceptible rats. A, Axial AIMs, comprised of trunk and neck dystonia, are not different between rAAV-Nurr1 and rAAV-GFP F344 rats. $B$, Orolingual AIMs, comprised of chewing and tongue protrusions, are not different between vector groups. C, Forelimb AIMs, comprised of forelimb hyperkinesia and dystonia, are not different at most time points between vector treatment groups. There was a significant difference between the groups at 150 min postinjection. ${ }^{*} p \leq 0.05$. Rating period shown at Day 17 with $8 \mathrm{mg} /$ $\mathrm{kg}$ L-DOPA dosing.

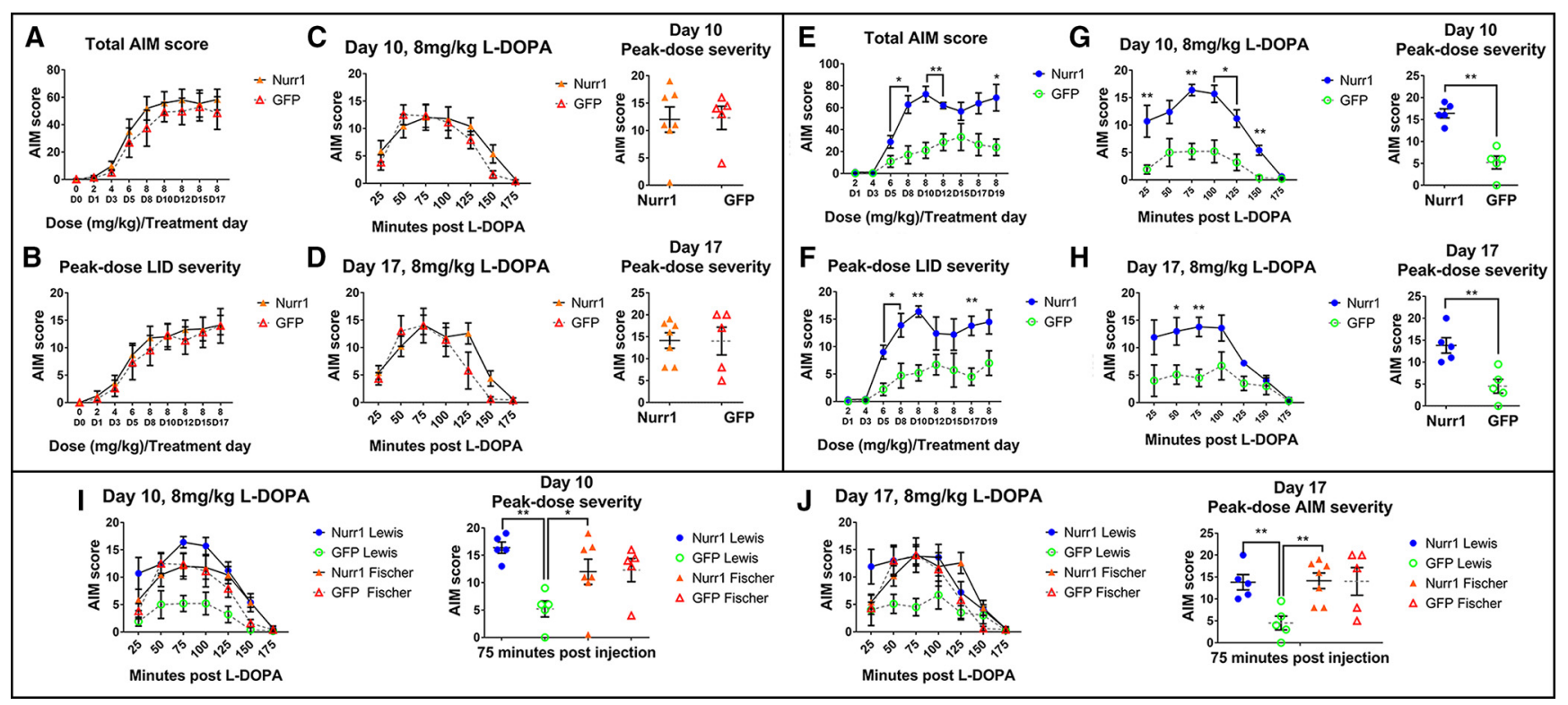

Figure 5. rAAV-mediated striatal overexpression of Nurr1 induces severe AIMs in LID-resistant rats. $\boldsymbol{A}$, The total AIM score sum of each rating session showed no differences between rAAVNurr1 and rAAV-GFP LID-susceptible F344 rats. Both groups developed severe AIMs similarly over the treatment regimen. $\boldsymbol{B}$, Peak-dose LID severity (75 min postinjection) over each rating period. No differences in peak-dose severity between vector groups was observed in F344 rats. C, D, Animals were treated with $8 \mathrm{mg} / \mathrm{kg}$ L-DOPA on Days 10-17. Both groups showed similar AlM expression over time (left) and peak-dose AIMs (right). $\boldsymbol{E}$, Total AIM score sum showed exacerbated AIMs on Days 5, 8, 10, 12, and 19 in rAAV-Nurr1 treated LID-resistant Lewis rats. $\boldsymbol{F}$, Peakdose LID severity show rAAV-Nurr1 treated Lewis rats developing significantly more severe AIMs than rAAV-GFP animals at 6 and $8 \mathrm{mg} / \mathrm{kg}$ doses. $\boldsymbol{G}, \boldsymbol{H}$, Individual rating periods on Days $10-17$ with $8 \mathrm{mg} / \mathrm{kg}$ treatment. rAAV-Nurr1 animals developed AIMs more severe than rAAV-GFP animals at multiple time points during the observation period. Days $10(\boldsymbol{I})$ and $17(\boldsymbol{J})$ AIM rating time course comparing rAAV treated LID-resistant Lewis rats to LID-susceptible F344 rats. rAAV-Nurr1 Lewis animals showed indistinguishable AIMs time course and peak-dose severity compared with both groups of F344 rats. rAAV-GFP Lewis animals showed significantly lower LID severity than both rAAV-Nurr1 treated Lewis and F344 rats. rAAV-GFP treated F344 rats did not show significantly higher AIMs than rAAV-GFP treated Lewis rats. ${ }^{*} p \leq 0.05,{ }^{* *} p \leq 0.01$.

The induction of severe AIMs with ectopic Nurr1 expression in an otherwise LID resistant strain shows that Nurr1 is directly involved in LID development.

Nurr1 agonist therapy exacerbates LID

Based on the evidence that ectopic striatalNurr1 expression can act as a molecular trigger for LID induction in otherwise resistant Lewis rats, we next sought to determine whether pharmacological activation of Nurr1, which is being investigated as a neuroprotective therapy for PD (Kim et al., 2015; Smith et al., 2015; Dong et al., 2016), would also exacerbate LID. Unilaterally parkinsonian SD animals were pretreated for 1 week with a neuroprotective dose of the Nurrl agonist AQ $(20 \mathrm{mg} / \mathrm{kg}$; Kim et al., 2015) followed by L-DOPA+AQ combined administration (Fig. $6 A$ ). AQ pretreatment, followed by daily administration of L-
DOPA + AQ showed statistical evidence of exacerbation of LID severity, which was evident across all doses (Fig. 6D) but being most notable compared with L-DOPA+Veh at the moderate $(6 \mathrm{mg} / \mathrm{kg})$ dose of L-DOPA (Fig. $6 B)$.

Nurr1 agonist therapy exacerbates low-dose RID

We next examined the impact of AQ on AIMs in unilaterally parkinsonian SD rats pretreated with $\mathrm{AQ}(20 \mathrm{mg} / \mathrm{kg})$ followed by chronic AQ plus ropinirole (Fig. $6 E$ ), a drug with less dyskinetic liability. Although less pronounced than the effect of $\mathrm{AQ}$ in the presence of L-DOPA, pretreatment, followed by daily administration of the same neuroprotective dose of AQ in the presence of ropinirole did reveal some statistical evidence of exacerbated RID severity (Fig. 6F,H). This was most evident at the higher dose of ropinirole $(0.5 \mathrm{mg} / \mathrm{kg})$ as reflected by a trend toward 
A

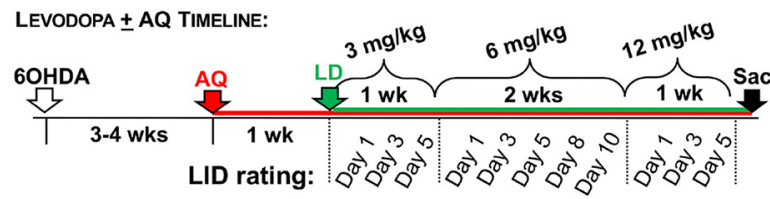

B

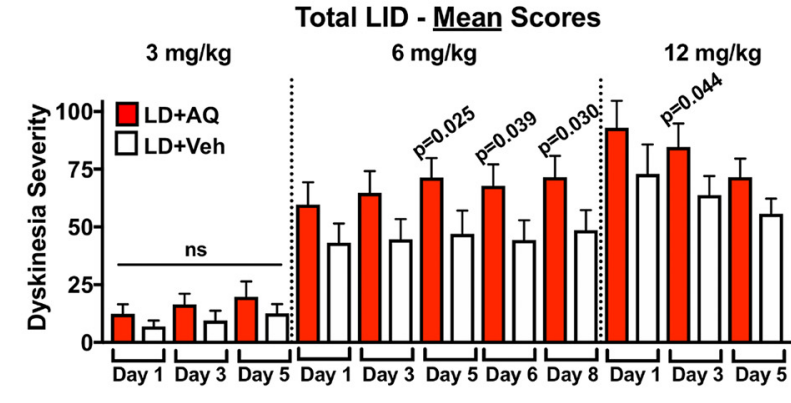

C

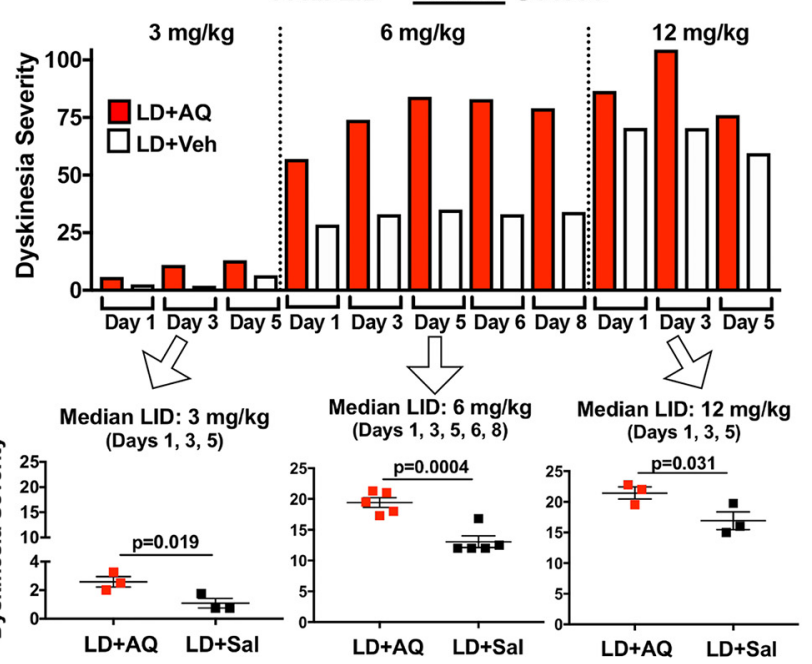

E

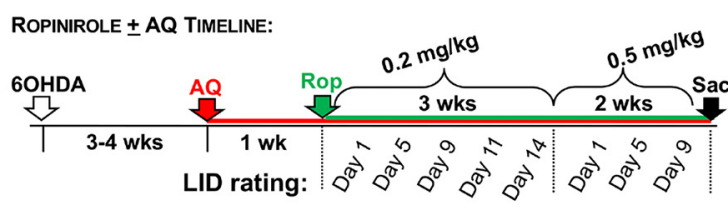

$\mathbf{F}$

Total RID - Mean Scores

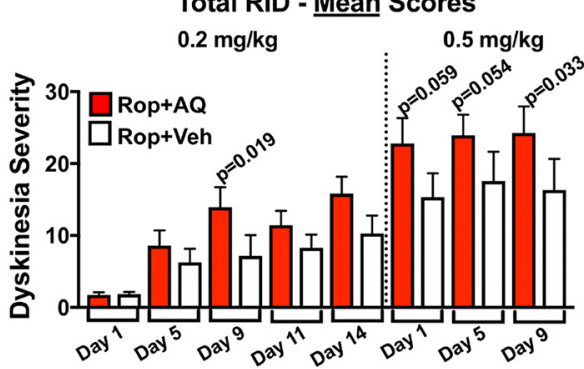

Total RID - Median Scores

G

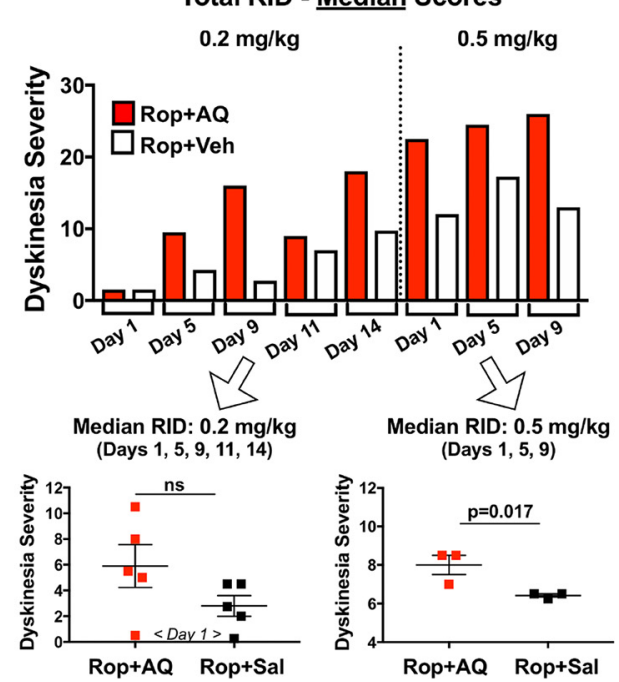

Figure 6. Neurobehavioral consequences of chronic Nurr1 agonist therapy on the expression of LID and RID in parkinsonian rats $A, E$, Timeline depicting treatment regimen with $A Q$ and L-DOPA $(\boldsymbol{A})$ or ropinirole $(\boldsymbol{E})$ in parkinsonian rats. $\boldsymbol{B}$, Mean LID AIM scores in parkinsonian SD rats were significantly higher in animals treated with L-DOPA + AQ compared with L-DOPA + Vehicle (Veh) at moderate $(6 \mathrm{mg} / \mathrm{kg})$ and high $(12 \mathrm{mg} / \mathrm{kg})$ doses of L-DOPA. C, D, Median scores show a pattern enhanced LID severity in L-DOPA + AQ compared with L-DOPA + Veh rats (C) with statistically enhanced severity when median scores are grouped by L-DOPA doses $(\boldsymbol{D})$. $\boldsymbol{F}$, Mean RID AIM scores show more modest evidence of enhanced dyskinesia severity in Ropinirole $(\mathrm{Rop})+\mathrm{AQ}$ compared with Rop + Veh groups, which was most evident at the higher dose $(0.5 \mathrm{mg} / \mathrm{kg})$ as reflected by a trend toward elevated mean RID severity at Days 1 and 3 , with significant difference at Day $9(\boldsymbol{F})$ together with the median data $(\boldsymbol{H})$. $\mathbf{G}$, Median RID AIM scores show a pattern enhanced LID severity in ropinirole + AQ compared with ropinirole + Veh rats. ns $=$ not significant.

elevated mean RID severity at Days 1 and 3 and significant difference at Day 9 (Fig. 6F), together with the median data (Fig. $6 H)$, which is less affected by outliers than mean data.

\section{Nurr1 expression is induced by pharmacological activation of direct pathway MSN}

We next examined whether preferential activation of either the striatal direct or indirect pathways is sufficient to reproduce the pathophysiological characteristics of LID and upregulation of Nurr1. Previous reports have found L-DOPA-induced Nurr1 expression in both direct pathway MSNs (dMSNs) and indirect pathway MSNs (iMSNs). To examine this, we induced AIMs in parkinsonian F344 rats with the selective D1/D5 agonist SKF81297 or D2/D3 agonist quinpirole; saline injection as a control. Rats were treated for 1 week with either drug and euthanized $2 \mathrm{~h}$ following the final dosing. Moderate and severe AIMs developed in animals treated with SKF-81297 (Fig. 7). Quinpirole-treated rats expressed significantly less severe AIMs. No saline-treated animals developed AIMs. Statistical analysis showed that drug-induced AIMs were significantly different between treatment groups (peak-dose AIMs: KruskalWallis statistic $=6.78, p<0.05$ ). IHC revealed abundant Nurr1 expression in the lesioned striatum of SKF-81 297 rats, but notably not in those expressing low-level AIMS following D2/ D3 agonist treatment or in the absence of AIMs. These data suggest that selective D1 receptor activation readily elevates Nurr1 and AIMs behavior, supporting the view that selective indirect pathway activation does not induce maladaptive striatal Nurr1 expression, but that direct pathway activation is required for this event.

To determine whether direct pathway activation leads to Nurr1 expression in predominantly dMSN, we performed dual label in situ hybridization with IHC to localize Nurr1 protein with mRNA of direct and indirect pathway markers (D1 and Enk, respectively; Lu et al., 1997). We observed cellular colocalization of Nurr1 protein and both markers of striatal projection neurons (D1 and Enk) in SKF-81 297 AIM-expressing animals (Fig. $7 F, G$ ). These findings suggest that Nurr1 expression in the indirect pathway is dependent on direct pathway activation. We also examined this in rats treated with quinpirole and saline, but 
A

AIM scores with DA agonists

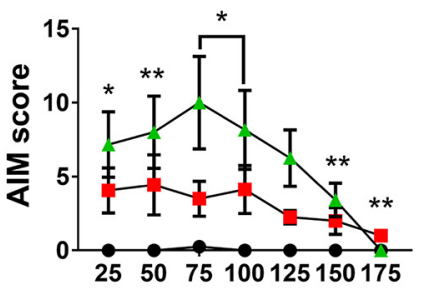

Minutes post L-DOPA
B

Peak-dose AIM severity

- Saline

- Quinpirole

t- SKF-81297

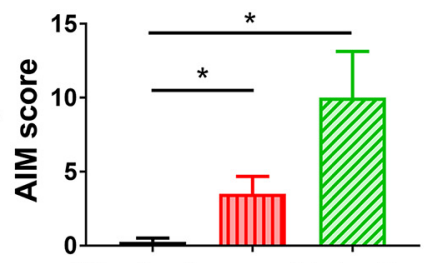

75 minutes post injection
Saline

III Quinpirole

SKF-81297

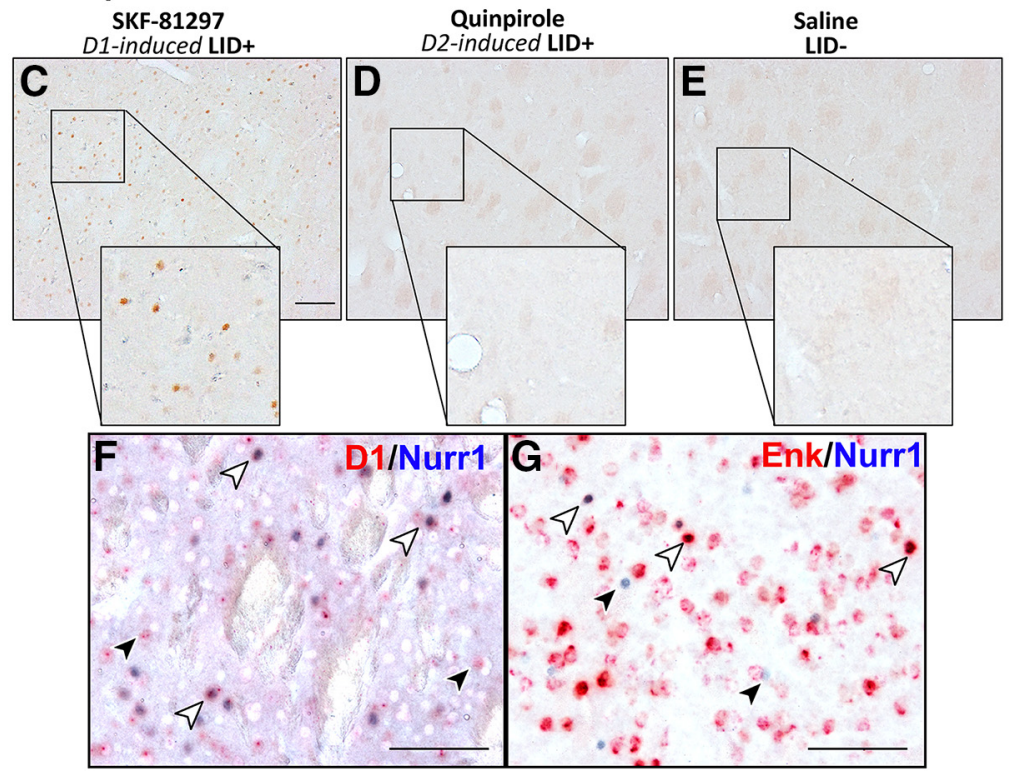

Figure 7. Abnormal striatal Nurr1 expression is induced by direct D1 receptor activation. A, AIM scores from the final treatment with either D1 agonist (SKF-81 297), D2 agonist (quinpirole), or saline. D1-agonist treated rats displayed severe AIMs, while D2 agonist treated animals expressed moderate AIMs. No AIMs were observed in saline treated animals. $\boldsymbol{B}$, Peak-dose AIM severity from the final drug treatment. ${ }^{*} p \leq 0.01,{ }^{* *} p \leq 0.05$. C $-\boldsymbol{E}$, Nurr1 IHC in lesioned striatum of animals treated with SKF-81 $297(\boldsymbol{C})$, quinpirole (D), or saline (E). Nurr1 protein was only observed in the lesioned striatum of dyskinetic rats treated with SKF-81 297 (C). Nurr1 was not observed in the lesioned striatum of LID+ rats treated with quinpirole $\boldsymbol{D}$ ). No Nurr1 was seen in saline treated animals (E). Scale bar, $100 \mu \mathrm{m}$. $\boldsymbol{F}$, In situ hybridization for D1 (red) and IHC for Nurr1 protein (blue) in striatum of LID+ animal treated with D1 agonist SKF-81 297. D1 transcript and Nurr1 protein are colocalized (white arrows) in some neurons, but not others (black arrows). G, In situ hybridization for enkephalin (red) and IHC for Nurr1 protein (blue) in the striatum of an LID+ animal treated with D1 agonist SKF-81 297. The enkephalin transcript is seen to colocalize with Nurr1 protein (white arrows). Some cells show Nurr1 expression with no enkephalin transcript (black arrows). Scale bar, $100 \mu \mathrm{m}$.

no colocalization was observed, because no Nurr1 protein was expressed in these animals (Fig. 8).

$r A A V$-induced Nurr1 expression in the absence of L-DOPA induces LID-like pathophysiological corticostriatal transmission and dendritic spine changes

Animals for electrophysiology and spine analysis studies were rendered parkinsonian with 6-OHDA injections as described previously (Schwarting and Huston, 1996; Soderstrom et al., 2010). Animals included in these analyses showed $\sim 75-80 \%$ cell loss in the injected hemisphere and there was no difference in lesion severity between vector groups (Fig. 9A-C; rAAV-Nurr1 \% TH neurons remaining $=21.48 \pm 3.12$; rAAVGFP \% TH neurons remaining $=27.87 \pm 5.26 ; t_{(13)}=1.08, p$ $>0.05)$. This degree of lesion is sufficient to induce enduring striatal changes that occur following DA depletion. Transgene expression was confirmed with IHC, and all animals included in the analysis showed robust transgene expression in the striatum (Fig. 9D,E). These animals were used for in vivo electrophysiology or spine analysis (the $N$ values for these studies are reported in the figure legends).

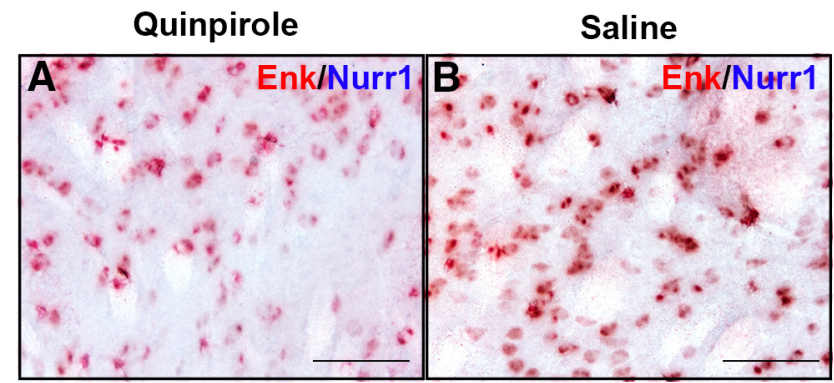

Figure 8. Nurr1 colocalization with iMSN in quinpirole and saline treated rats $\boldsymbol{A}$, In situ hybridization for enkephalin (Enk) and with IHC for Nurr1 in a dyskinetic rat treated with quinpirole. No Nurr1 protein was observed in these animals. $\boldsymbol{B}$, In situ hybridization for Enk with IHC for Nurr1 in a saline treated animal show no abnormal Nurr1 induction in saline treated rats.

To understand how ectopic Nurr1 expression can impact corticostriatal signaling and striatal output, we performed in vivo extracellular single-unit recordings of MSNs while testing for orthodromic and antidromic responses, before and after an acute saline or L-DOPA challenge. These studies yielded recordings 


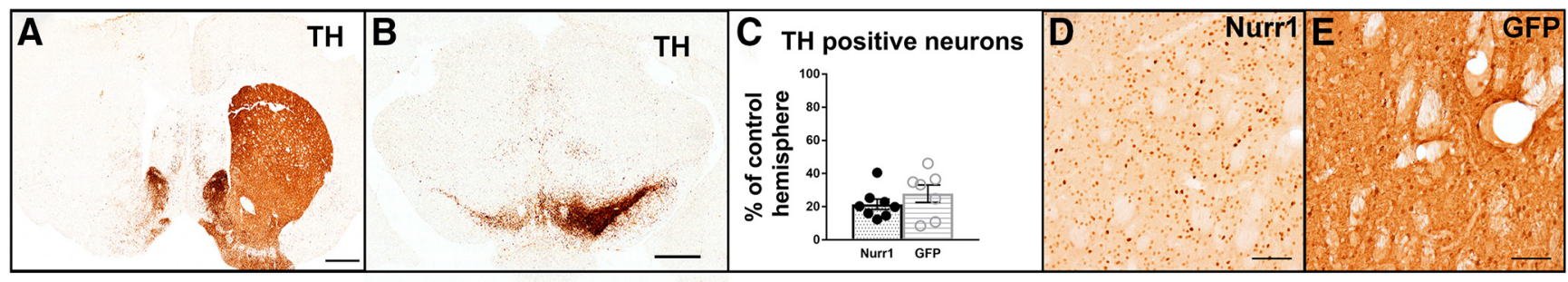

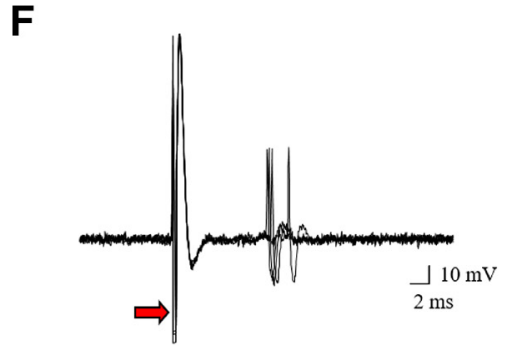

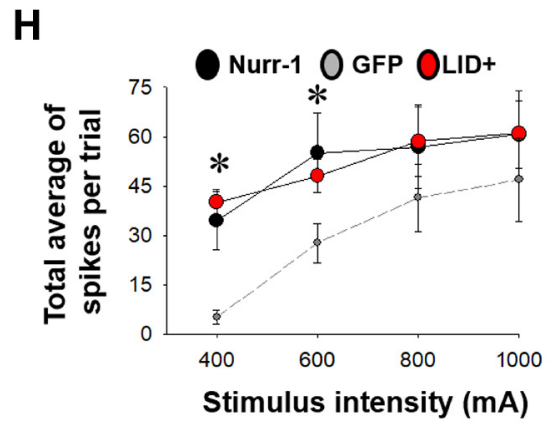

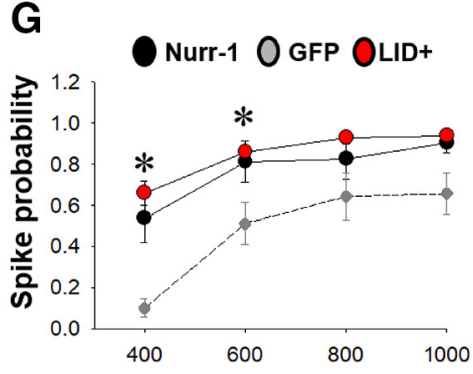

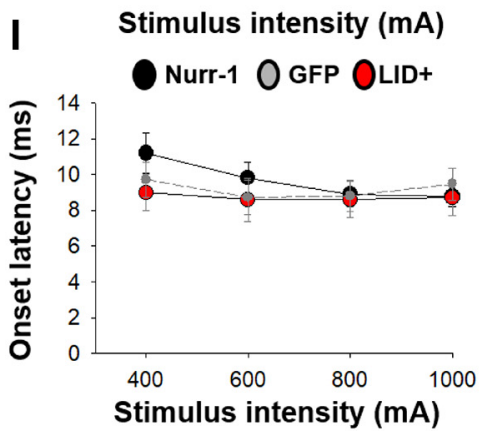

$\mathbf{J}$

\section{Current threshold}

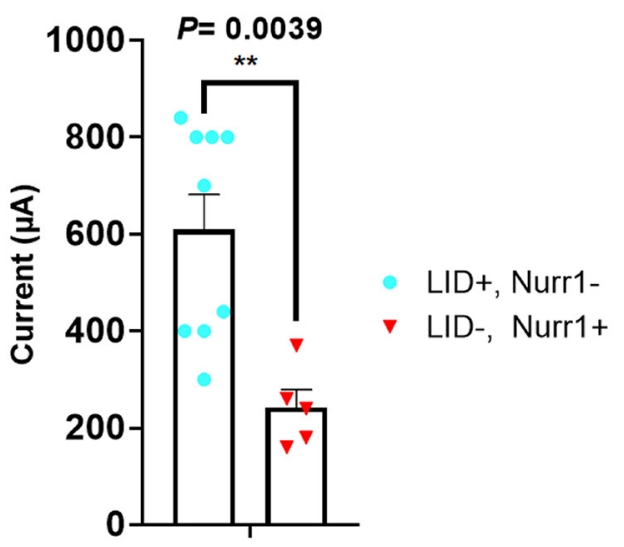

Figure 9. Comparisons between cortically-evoked spike characteristics of antidromically-identified striatonigral projection neurons recorded from DA depleted rAAV-naïve rats treated with chronic L-DOPA (LID+), rAAV-GFP injected L-DOPA naïve animals, or rAAV-Nurr1 injected L-DOPA naïve animals. A-C, Lesion status was confirmed with IHC for TH. TH immunoreactivity was dramatically reduced in the interjected hemisphere of the striatum $(\boldsymbol{A})$ and substantia nigra $(\boldsymbol{B})$. Stereological estimates of remaining TH-positive neurons show significant cell loss in both vector groups (C). D, E, Transgene expression from viral vector delivery was confirmed in the striatum with IHC for Nurr1 (D) or GFP (E). Scale bars: $\boldsymbol{A}, \boldsymbol{B}, 1 \mathrm{~mm} ; \boldsymbol{D}, \boldsymbol{E} 1 \mu \mathrm{m}$. $\boldsymbol{F}-\boldsymbol{J}$, Comparisons between cortically-evoked spike characteristics of antidromically-activated striatonigral projection neurons recorded from DA-depleted rAAV-naive rats treated with chronic L-DOPA (LID+), rAAV-GFP injected L-DOPA naive animals, or rAAV-Nurr1 injected L-DOPA naive animals. $F$, Traces of typical cortically-evoked responses from isolated striatonigral projection neurons. Ten consecutive overlaid responses are shown. Graphs compare the spike probability $(\boldsymbol{G})$, average number of total spikes evoked during a stimulus trial at each current intensity tested $(\boldsymbol{H})$, and onset latency of cortically-evoked spikes, in antidromically-identified dMSNs during cortical stimulation ( $)$. Main stimulus intensity-dependent effects on cortically-evoked spike probability of dMSNs (striatonigral projection neurons) were observed in both LID + and Nurr-1 overexpressing rats compared with vehicle treated DA-depleted GFP-expressing controls $\left({ }^{*} p<0.001\right)$. Post hoc comparisons revealed a significant increase in the probability and number of evoked responses to cortical stimulation at the 400-600 $\mu$ A current intensities in both LID + and Nurr-1 overexpressing rats compared with vehicle treated DA-depleted, GFP-expressing control rats $\left({ }^{*} p<0.05\right)$. No significant differences in onset latency ( $p>0.05$ ) or SD of latency (data not shown) of corticallyevoked responses were observed. $J$, The current threshold for eliciting antidromic spike activity in identified striatonigral dMSNs, which were not responsive to cortical stimulation recorded in rAAV-Nurr1 rats $(242 \mu \mathrm{A} \pm 36.9)$ was significantly lower than that of vector naive, LID + rats $(608.9 \mu \mathrm{A} \pm 72.9, p=0.0039)$, indicating that Nurr1 overexpression leads to an L-DOPA-independent upregulation in the axonal/terminal excitability of dMSNs that is greater than that induced by chronic L-DOPA exposure. Data are derived from N=9/6 D1+ Nurr1- DA-depleted cells/ rats (saline $+\mathrm{L}-\mathrm{DOPA} ; 5 \mathrm{mg} / \mathrm{kg}$ chronic, same acute challenge dose on the recording day), and $N=5 / 4$ Nurr1 + DA-depleted cells/rats (saline $+\mathrm{L}-\mathrm{DOPA} ; 5 \mathrm{mg} / \mathrm{kg}$ acute challenge). ${ }^{* *} p=$ 0.0039 .

from both identified dMSNs (antidromically activated from the SNr; i.e., SNr+; Fig. 9), as well as a larger population of unidentified MSNs likely composed of dMSNs as well as iMSNs (SNr-; Fig. 10).

All MSNs that were not successfully identified $(\mathrm{SNr}-)$ were combined together and compared across groups to gain insight into possible changes in striatal output as a whole (Fig. 10), in LDOPA naive parkinsonian rats injected with rAAV-Nurr1 or rAAV-GFP. After a MSN was isolated, spontaneous and cortically-evoked responses to a range of stimulation intensities $(1000,800,600$, and $400 \mu \mathrm{A})$, and the presence of antidromic responses were recorded as described above and in previous studies (Threlfell et al., 2009).

First we compared the spike probability and onset latency of cortically-evoked spikes in identified $(\mathrm{SNr}+) \mathrm{dMSN}$ recorded from DA-depleted rAAV-naive rats treated with chronic L-
DOPA (LID+), rAAV-GFP injected L-DOPA naïve animals, or rAAV-Nurr1 injected L-DOPA naïve animals (Fig. 9F-I). We were able to positively identify a subpopulation of dMSNs via antidromic stimulation in all three groups. In this population of rAAV-Nurr1 rats, cortically-evoked spike responses were found to mimic typical activity observed in $\mathrm{AAV}$-naïve dyskinetic rats, compared with nontreated vector control rats which showed a much less robust response to cortically stimulation (Fig. 9G,H). Last, we measured the current threshold required to elicit antidromic activation in identified $\mathrm{dMSN}$ s that did not respond to cortical stimulation but did respond to antidromic activation $(\mathrm{SNr}+)$, in vector naïve, $\mathrm{LID}+$ rats and compared them to similar measures from non-dyskinetic rats treated with rAAV-Nurr1. We found that in the striatum of rAAV-Nurr1 rats, dMSNs exhibit a significant reduction in the current threshold required to elicit antidromic spike activity compared with dMSNs recorded 

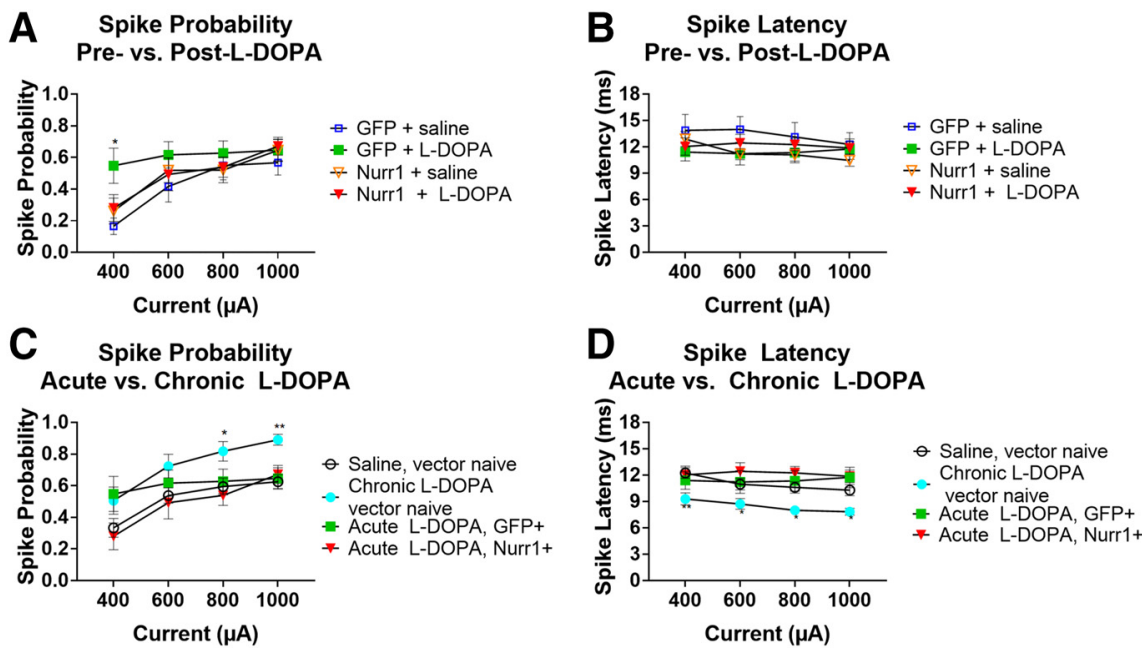

Figure 10. Comparisons between cortically-evoked spike characteristics of unidentified MSNs recorded from DAdepleted parkinsonian, vector naive rats treated with saline or chronic L-DOPA, and vector-treated (rAAV-GFP or rAAVNurr1) animals not chronically treated with L-DOPA. A-D, Cortically-evoked spike probability pre- and post-L-DOPA delivery in GFP and Nurr1 injected rats. MSNs recorded in GFP rats showed a higher spike probability after L-DOPA delivery at $400 \mu \mathrm{A}$ stimulation ( $n=12-21$ cells, 7-10 rats per group). $\boldsymbol{B}$, No differences in the onset latency of cortically-evoked spikes was observed in either vector group before, and following L-DOPA injection ( $n=12-23$ cells, 7-10 rats per group). C, MSNs recorded in established LID+ rats (chronic L-DOPA, vector naive) exhibited higher cortically-evoked spike probability than those recorded in GFP injected, Nurr1 injected, and rAAV-naive saline treated controls ( $n=13-31$ cells, 7-16 rats per group). $D$, LID + rats exhibited significantly shorter spike onset latencies than both vector-treated groups and saline controls ( ${ }^{*} p \leq 0.05,{ }^{* *} p \leq 0.01 ; n=12-32$ cells, $7-16$ rats per group).

in animals with established dyskinesias (rAAV-Nurr1 L-DOPA naïve, $242 \mu \mathrm{A} \pm 36.9 ; \quad$ rAAV-naïve $\mathrm{LID}+, \quad 608.9 \mu \mathrm{A} \pm 72.9$; $p=0.0039$; Fig. 9J). Together, these studies indicate that dMSNs ectopically expressing Nurr1 are significantly more responsive to excitatory corticostriatal synaptic drive, and at least a subpopulation of dMSNs, which could not be driven by cortical stimulation, exhibit enhanced axonal/terminal membrane excitability compared with dMSNs recorded in LID + rats. Thus, Nurr1 may be priming the abnormal signaling activity that occurs with chronic L-DOPA therapy associated with LID.

We next compared vector treated rats with vector naïve animals treated chronically with L-DOPA, and therefore LID+. Spike probability was increased, and spike onset latency was decreased in LID + animals, indicating an increase in corticostriatal drive onto MSNs following chronic L-DOPA exposure. Both the spike probability and onset latency of cortically-evoked spikes recorded in rAAV-GFP and rAAV-Nurr1 animals were significantly different compared with similar measures performed in LID + animals, and these responses more resembled vector naïve, L-DOPA naïve rats (Fig. 9H,I). These outcomes suggest that pathophysiological alterations in corticostriatal circuits affecting striatal projection neuron activity are dependent on LID presence and not Nurr1 expression alone.

Finally, we measured the current threshold required to elicit antidromic activation in identified dMSNs in vector naïve, LID+ rats and compared them to similar measures from non-dyskinetic rats treated with rAAV-Nurr1. We found that in the striatum of rAAV-Nurr1 rats, dMSNs exhibit a significant reduction in the current threshold required to elicit antidromic spike activity in dMSNs compared with animals with established dyskinesias (rAAV-Nurr1 L-DOPA naïve, $242 \mu \mathrm{A} \pm 36.9$; rAAV-naïve $\mathrm{LID}+, 608.9 \mu \mathrm{A} \pm 72.9 ; p=0.0039$; Fig. $9 J)$. This indicates that dMSNs ectopically expressing Nurr1 are significantly more responsive to excitatory corticostriatal synaptic drive, suggesting
Nurr1 may be priming the abnormal signaling activity that occurs with chronic LDOPA therapy associated with LID.

\section{MSN spine density and morphology} changes are induced by Nurr1 expression Dramatic changes in spine density and morphology in LID models has been previously demonstrated (Zhang et al., 2013; Fieblinger and Cenci, 2015). In addition, Nurr1 is involved in learning and memoryassociated plasticity classically associated with spine changes (Pena de Ortiz et al., 2000; Colon-Cesario et al., 2006). Given that LID are often considered an aberrant form of motor learning we sought to determine whether Nurr1 expression could affect MSN spines in the absence of exposure to L-DOPA. Similar to the rats used for electrophysiology, these animals did not receive L-DOPA at any time, again allowing us to determine the effect of Nurr1 on DA-depleted MSNs independent of drug treatment. Spine analysis revealed there was a significant decrease in total spine density in rAAV-Nurrl treated animals (rAAV-Nurrl total spines/ $10 \mu \mathrm{m}=4.85$ $\pm 0.10, \mathrm{rAAV}-\mathrm{GFP}$ total spines $/ 10 \mu \mathrm{m}=$ $5.98 \pm 0.31 ; \quad t_{(5)}=3.68, p<0.05 ;$ Fig. $11 A)$. This difference was not specific to either the proximal or distal portions of the dendrite (rAAV-Nurr1 proximal spines $/ 10 \mu \mathrm{m}=3.54 \pm 0.31$, distal spines $/ 10 \mu \mathrm{m}=5.58 \pm 0.41$; rAAV-GFP proximal spines $/ 10 \mu \mathrm{m}=4.24 \pm 1.45$, distal spines/ $10 \mu \mathrm{m}=6.89 \pm 0.74)$. These data clearly indicate that Nurr1 does impact striatal dendritic spine dynamics, even in the absence of DA signaling related to L-DOPA, and may play a role in spine plasticity changes accompanying LID.

We next compared spine morphology, an indicator of synaptic strength and spine dynamics (Maiti et al., 2015), between rAAV-Nurr1 and rAAV-GFP animals. We found that ectopic Nurr1 expression led to a change in two specific spine phenotypes. rAAV-Nurr1 animals displayed significantly fewer thin spines than controls (rAAV-Nurrl thin spines $/ 10 \mu \mathrm{m}=3.38 \pm$ 0.09 , rAAV-GFP thin spines $/ 10 \mu \mathrm{m}=4.48 \pm 0.43 ; t_{(5)}=2.98$, $p<0.05$ ). We also observed fewer branched (also known as cupped or bifurcated) spines in rAAV-Nurr1 (branched spines/ $10 \mu \mathrm{m}=0.15 \pm 0.01$ ) than in rAAV-GFP animals (branched spines $/ 10 \mu \mathrm{m}=0.23 \pm 0.02 ; t_{(5)}=3.35, p<0.05$; Fig. $\left.11 B\right)$. No difference between groups was observed with stubby or mushroom spines. Cumulatively, these data implicate Nurr1, independent of L-DOPA, as a molecular regulator of the maladaptive striatal spine plasticity that has been shown in animal models of LID.

\section{Nurr1 is expressed in the striatum of $L-D O P A$-treated PD} patients

Conservation of a variable across species is important to support not only its involvement in preclinical models but its relevance to the human disease state. In order to begin to assess whether Nurr1 induction also occurs in human disease, we performed a small qualitative IHC study to assess Nurr1 expression in the striatum of dyskinetic PD patients who had been treated with LDOPA. In multiple cases (all confirmed dyskinetic), we observed 

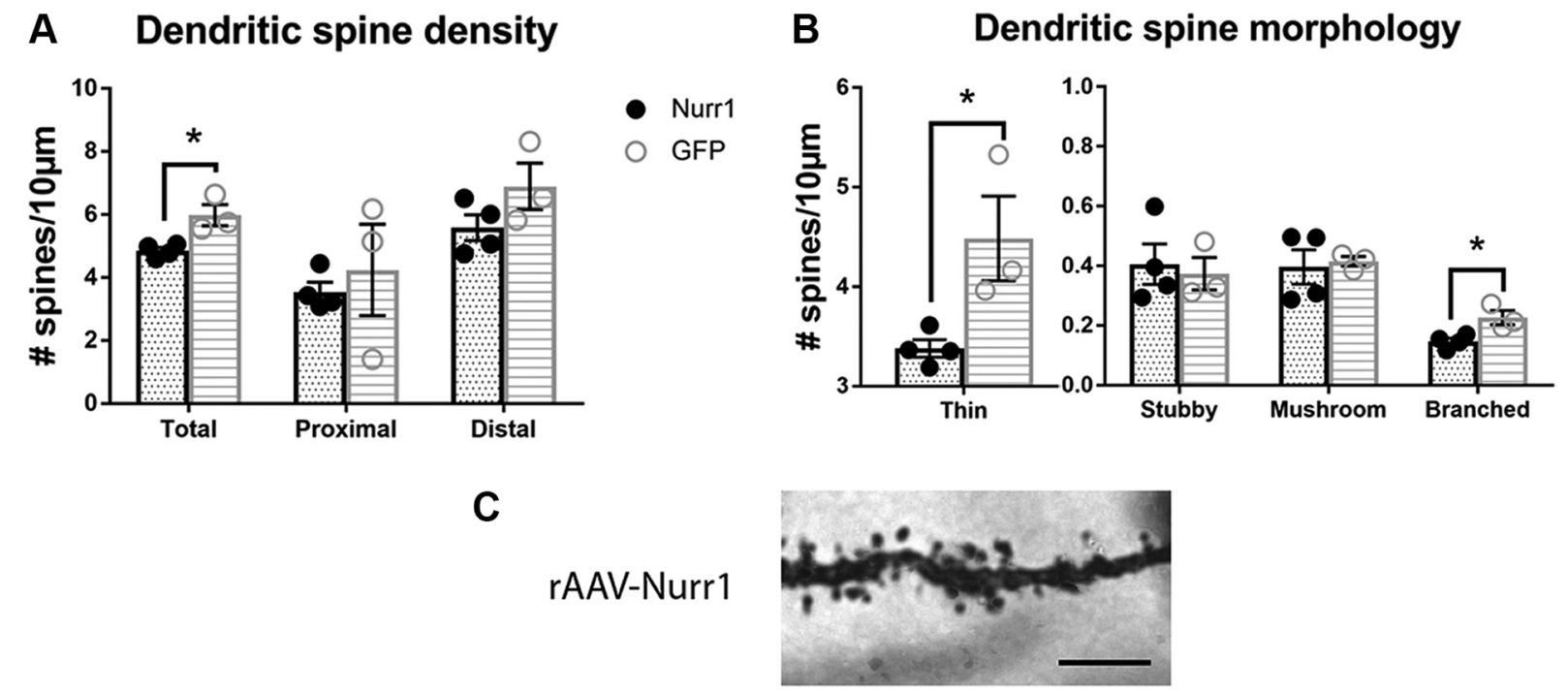

rAAV-GFP

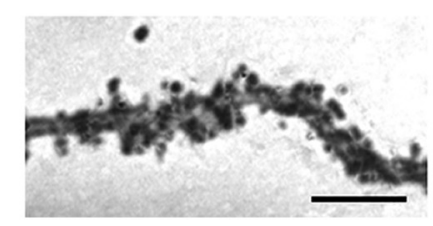

Figure 11. Nurr1-induced alterations in dendritic spine density and morphology $A$, Dendritic spine density of MSNs in the lesioned hemisphere of rats treated with rAAV-Nurr1 or rAAV-GFP. Nurr1 expression caused a decrease in total number of spines. rAAV-Nurr1 total spines $/ 10 \mu \mathrm{m}=4.85 \pm 0.10$, rAAV-GFP total spines $/ 10 \mu \mathrm{m}=5.98 \pm 0.31 ; t_{(5)}=3.68,{ }^{*} p \leq 0.05 . \quad B$, rAAVNurr1 expression induced a decrease in both thin and branched type spines compared with rAAV-GFP controls. rAAV-Nurr1 thin spines/10 $\mu \mathrm{m}=3.38 \pm 0.09$, rAAV-GFP thin spines/ $10 \mu \mathrm{m}=4.48 \pm 0.43 ; t_{(5)}=2.98,{ }^{*} p \leq 0.05$. C, Representative image of dendritic spines on Golgi-Cox stained MSN. Scale bar, $50 \mu \mathrm{m}$.

distinct nuclear Nurr1 staining in the putamen of PD patients (Fig. 12A). This signal was not observed in age-matched controls with DLB that never received L-DOPA (Fig. 12B). This observation is the first direct connection of Nurr1 to LID in PD patients. It is important to note that the scope of this analysis was limited in terms of the number of patients. Moreover, the extremely limited availability of postmortem tissue from L-DOPA treated, LID negative, subjects precluded us from assessing the status of striatal Nurr1 in this crucial control group.

\section{Discussion}

Although previous studies have demonstrated that ectopic induction of striatal Nurr1 is associated with LID (Heiman et al., 2014; Sodersten et al., 2014), the current study demonstrates that not only is striatal Nurr1 expression capable of inducing LID behavior in genetically resistant subjects, but also provides the first evidence of a genetic modifier of LID directly inducing morphologic and electrophysiological signatures of this behavioral malady independent of L-DOPA. In the following paragraphs we provide discussion related to the contribution of our studies in advancing understanding of the role of this transcription factor in LID, as well as potential clinical ramifications.

Genotype-to-phenotype impact of ectopic striatal Nurr1

The homogenous genotype of inbred rat strains has served in preclinical models to advance understanding genetic factors underlying differences in behavioral phenotypes. Lewis and F344 rats are well known to display differences in response to psychostimulant addiction liability (Brodkin et al., 1998; Miguens et al., 2011; Valenza et al., 2016; Fole et al., 2017), a behavioral phenomenon with many similarities to LID in that both are considered to result from aberrant associative or motor learning and rewiring of basal ganglia neural circuits following DA-related priming. We recently demonstrated in an extensive characterization study (Steece-Collier et al., 2020) that these rat strains also show dramatically different LID liability, with Lewis rats showing distinct resistance to LID expression in contrast to robust development in F344 rats. Using this novel model, we report here that ectopic induction of striatal Nurr1 in LID-resistant Lewis rats can induce LID severity to the same high level as seen in LIDprone F344 rats, suggesting that striatal Nurr1 expression is a key factor in: (1) LID development, and (2) the differential susceptibility between these two inbred rat strains. Further, these data suggest that Nurrl expression is not a downstream byproduct of LID, but rather plays a causative role in LID development. Continuing studies aimed at identifying genetic or epigenetic differences between the strains that may explain their differential upregulation of Nurr1 in response to L-DOPA would be valuable for the identification of molecular traits or signatures involved in LID susceptibility.

In addition to ectopic induction of striatal Nurr1 being capable of converting genetically LID-resistant rats to LID-expressing rats, the current data provides novel insight that this particular genetic modifier of LID can directly induce striatal phenotypic morphologic and electrophysiological changes independent of LDOPA. Specifically, we found that ectopic Nurr1 expression in the absence of L-DOPA resulted in a decrease in total number of dendritic spines, reflective of specific decreases in thin and branched spines.

Although there remains some controversy as to the exact nature of spine changes in the parkinsonian striatum, it is well established that striatal DA depletion results in loss of dendritic spines on MSNs in PD and in animal models of PD (McNeill et al., 1988; Ingham et al., 1989; Day et al., 2006; Soderstrom et al., 

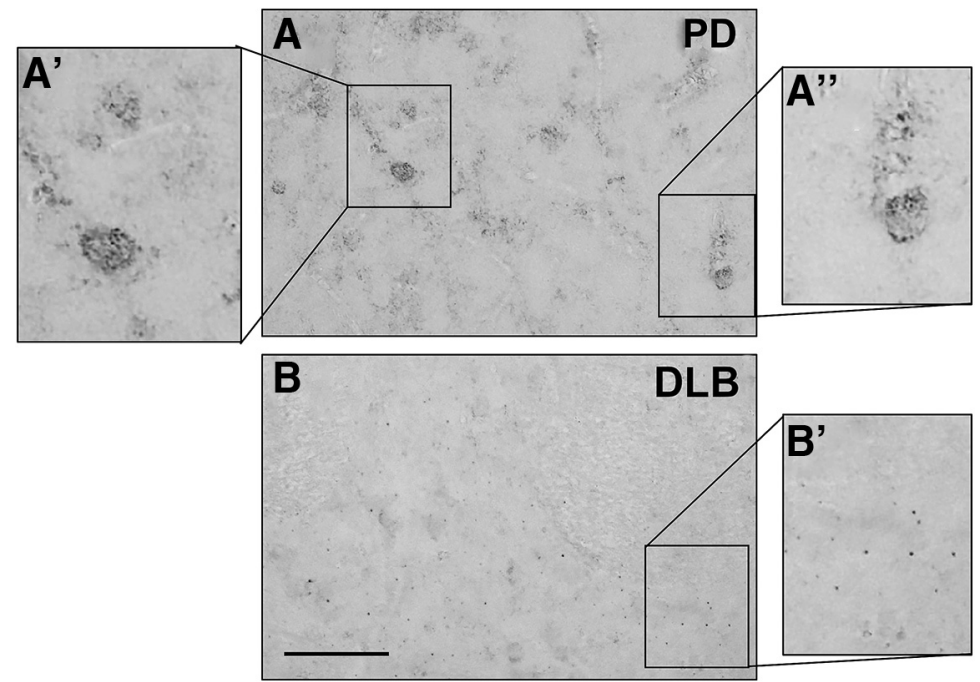

Figure 12. Nurr1 is expressed in the striatum of L-DOPA treated PD patients. IHC for Nurr1 in the putamen of postmortem patient samples of a Parkinson's disease patient diagnosed with LID $(\boldsymbol{A})$ and an age-matched patient with DLB (LDOPA naive). Areas in $\boldsymbol{A}^{\prime}$ and $\boldsymbol{A}^{\prime \prime}$ show areas of increased magnification in $\boldsymbol{A}$ (Nurr1 + ). Area in $\boldsymbol{B}^{\prime}$ shows Nurr1-negative area outlined in $\boldsymbol{B}$. Scale bar, $50 \mu \mathrm{m}$.

2010; Zhang et al., 2013; Fieblinger et al., 2014; Nishijima et al., 2014; Fieblinger and Cenci, 2015), with dyskinogenic L-DOPA generally restoring spine density (for details of this complex phenomenon, see Bastide et al., 2015). Thus, although a decrease in total spine number following ectopic Nurrl induction may appear contrary to the previous reports of an overall restoration in the striatal spine density in dyskinetic animals, studies in both mice and rats (Zhang et al., 2013; Fieblinger et al., 2014; Suarez et al., 2014) have reported that despite some degree of re-establishment of spines with LID, the total density tends to remain statistically below that seen in intact subjects (Zhang et al., 2013; Fieblinger et al., 2014). Specifically, Fieblinger et al. (2014) demonstrate in transgenic (tg) mice with green fluorescent protein or tdTomato under control of D1 or D2 receptor regulatory elements, which allows specific examination of iMSNs and dMSNs, that DA depletion resulted in loss of dendritic spines only in iMSNs but not dMSNs. In the presence of dyskinogenic L-DOPA they found that even when iMSNs showed a restoration of spines density, the total number of spines remained below levels in the intact striatum. And this occurred coincident with dMSNs showing a further significant decrease in total number of spines compared with both control and parkinsonian striatum. In contrast Suarez et al. (2014) found that spine density in these same parkinsonian tg mice was decreased in both iMSNs and dMSNs, but similar to Fieblinger et al. (2014) they too found that in the dyskinogenic striatum spine density was (1) restored to control levels in iMSNs, (2) significantly reduced in dMSNs, and (3) overall total spine density was less than in sham controls. In addition, we have previously demonstrated using Golgi methods that in LDOPA treated, LID expressing parkinsonian rats, that there is a significant reduction in spine density compared with the nonlesioned control rats (Zhang et al., 2013). Although our previous data showed a trend for total spine density in dyskinetic rats to be higher than in non-L-DOPA-treated parkinsonian rats, perhaps related to the aforementioned differential iMSN versus dMSN spine dynamics, dyskinogenic and drug naïve parkinsonian rats all show decrease in spine density compared with intact control striatum (Zhang et al., 2013). Although the Golgi impregnation methods used here do not allow us to identify whether one or both populations of MSNs was/were affected by Nurr1 overexpression, our data clearly provide the first evidence that striatal Nurr1, in the absence of L-DOPA, does impact dendritic spine dynamics in a similar fashion as reported with LID (Zhang et al., 2013; Fieblinger et al., 2014; Suarez et al., 2014). Indeed, although we report that ectopic Nurr1 expression results in a decrease in spine density in the parkinsonian striatum it is uncertain whether this was due to an unintended biased sampling of direct pathway over indirect pathway MSNs, or was related to the possibility that ectopic striatal Nurr1 in the absence of non-physiological DA stimulation causes a generalized decrease in spine density. Regardless, together the present data support the idea that striatal Nurr1 impacts spine plasticity and implicates this orphan nuclear receptor as a molecular factor involved in promoting the maladaptive spines changes associated with LID.

Although the mechanism of this effect remains to be determined, Nurr1 is known to heterodimerize with another NR4A transcription factor, Nur77 (Maira et al., 1999). Nur77 is endogenously expressed in the adult striatum, and is increased by LDOPA treatment in denervated animals, primarily in dMSNs (StHilaire et al., 2003; Mahmoudi et al., 2009). Similar to our findings, viral overexpression of Nur77 in the striatum can exacerbate AIMs in rats (Rouillard et al., 2018) and also induce spine loss in hippocampal neurons, supporting an important role the NR4A family of transcription factors in affecting synaptic plasticity (Chen et al., 2014).

In keeping with ectopic Nurr1 directly affecting corticostriatal synaptic plasticity we show here that Nurr1 expression alone, independent of dyskinesiogenic doses of L-DOPA, can induce an LID-like alteration in cortically-evoked firing specifically in dMSNs responding to an to acute, non-dyskinesiogenic, lowdose of L-DOPA given at the time of recording. Specifically, these studies provide novel evidence that ectopic expression of Nurr1 is capable of inducing hyper-corticostriatal drive and increased output from dMSNs. Indeed, in a small subset of identified dMSNs (Fig. 9), we saw an identical increase, compared with rAAV-GFP-treated subjects, in spike probability in rAAVNurr1-expressing animals which was similar to that observed in LID + rats treated chronically with L-DOPA, suggesting that Nurr1 induces an enhancement in excitatory corticostriatal synaptic plasticity preferentially in dMSNs, regardless of chronic LDOPA administration and LID expression. This increase in responsiveness to cortical drive is consistent with the above observed decrease in spine density as this would be expected to result in a more electrotonically compact neuronal membrane due to the resulting increase in MSN membrane resistance, leading to increased intrinsic excitability and potentially, larger postsynaptic responses to excitatory glutamatergic corticostriatal drive (Gertler et al., 2008).

Direct pathway activation is required for Nurr1 induction Although there is considerable evidence for interactions between the direct and indirect pathways in LID pathology, Nurr1 has 
previously been reported to be expressed primarily in MSNs of the direct pathway in association with LID (Heiman et al., 2014). Our pharmacological studies using the selective D1/D5 agonist SKF-81 297 or D2/D3 agonist quinpirole support that although AIMs are observed under conditions of either preferential direct or indirect pathway activation, AIMs are more severe with a selective D1 receptor agonist and Nurr1 induction in striatal MSNs occurred only in parkinsonian rats treated with the DA D1 receptor agonist. Interestingly, despite preferential activation of D1 receptor MSN, we observed Nurr1 induction in both the direct and indirect pathway MSNs. It is uncertain why Nurr1 transcript was not induced following treatment with the selective D2 receptor agonist. It is possible that there are D2-dependent, Nurr1-independent pathways that can lead to LID development, suggesting that the mechanism for the low-severity LID development in the Lewis strain is a distinct phenotype from the canonical parkinsonian LID. However, although AIMs were observed with quinpirole, the phenotype was not only less severe, but was also generally more reflective of hyperkinesia than the trunk and limb dystonia typical of SKF-81 297 or L-DOPA.

These findings indicate that Nurrl induction in the striatum is dependent on supra-physiological activation of striatonigral dMSN transmission, however, this may not be completely determined in a singular manner by increased excitatory corticostriatal drive (i.e., changes in inhibitory influences driven by striatal fast-spiking interneurons may also be occurring, which could shunt cortical drive, despite the concurrent expression of increased intrinsic membrane excitability). Based on our previous studies characterizing the excitatory effect of the phosphodiesterase 10A inhibitor compound TP-10 (increase in proportion of striatonigral/dMSNs responding to antidromic activation; no change in cortically-evoked spikes in these same cells; (Threlfell et al., 2009), and other studies (Padovan-Neto et al., 2015; Beaumont et al., 2016), it is plausible that the large decreases in antidromic threshold observed herein may point to increases in axonal membrane or terminal excitability at the level of the midbrain, induced by Nurr-1 overexpression in dMSN. Furthermore, these changes in intrinsic membrane excitability can be indirectly inferred or detected by this antidromic activation/terminal excitability approach (Groves, 1983). Based on the above studies, we know that excitatory increases in MSN membrane activity can occur in Huntington's disease models which are opposite to the deficit in excitatory corticostriatal drive observed in these models (Cepeda et al., 2007; Sepers and Raymond, 2014; Beaumont et al., 2016; Bunner and Rebec, 2016). It is likely that the intrinsic membrane excitability may increase and become more responsive to local current injection, and as thought in HD, in a compensatory manner as a result of decreased afferent drive, causing changes in the balance between inhibitory and excitatory afferent inputs at the level of the cell body (e.g., striatum) could limit cortically-driven synaptic activity in a manner that is compartmentalized in the distal dendrites, although changes in plasticity can also occur at the level of axonal membrane that would not be affected by the striatal afferents.

Additionally, our studies indicate that D1 receptor activation is sufficient to induce Nurr1 expression in not only direct, but also a subpopulation of iMSNs. These data align with studies of other immediate early genes in LID, such as Homer-1a and FosB, which show that D1 priming is required for expression in D2 neurons (Pollack and Yates, 1999; Yamada et al., 2007; Pollack and Thomas, 2010). Based on the finding that F344 rats treated with the D2 selective agonist quinpirole and Lewis rats treated with L-DOPA develop only mild-to-moderate AIMs, which were not associated with striatal Nurr1 induction, it is reasonable to suggests that Nurr1 may act as a "master regulator" in the striatum and when expressed/activated can induce changes in corticostriatal transmission, striatal output, and motor behavior that lead to more severe dyskinesia.

\section{A mechanistic model of Nurr1 activation}

In recent work we demonstrated that genetic silencing of striatal Cav1.3 L-type channel in hemi-parkinsonian rats both prevents and reverses LID (Steece-Collier et al., 2019). Cav1.3 has long been appreciated as a key regulator of dendritic spine morphology, including that of MSN, and loss of striatal DA is linked to dysregulation of this channel (Day et al., 2006). Downstream effects of activation of L-type calcium channels are linked to gene regulation and neuronal plasticity (Stanika et al., 2016). However, the mediators of this activity remain elusive. To that end, our data presented herein suggests that Nurr1 may be an important meditator of Cav1.3 transcriptional and plasticity activity. Indeed, in vitro studies have shown that Cav1.3 activity, mediated via calcineurin, can result in induction of Nurr1 expression (Tokuoka et al., 2014). As discussed elsewhere, depending on the level of expression (Johnson et al., 2011; Chen et al., 2014), this induction in Nurr1 expression would thus be a chief modulator of dendritic spine changes. Further work is needed to fully elucidate effector molecules downstream of Nurr1 transcriptional activity.

\section{Clinical indications for Nurr1 in PD: Nurr1 agonists and LID}

Nurr1 has received a great deal of attention in the past several of years for its essential role in the survival of nigral DA neurons (Zetterstrom et al., 1997; Jiang et al., 2005; Kadkhodaei et al., 2009), and its potential to slow death of these neurons in PD (Decressac et al., 2013; De Miranda et al., 2015; Kim et al., 2015; Smith et al., 2015; Volakakis et al., 2015; Dong et al., 2016). However, although Nurr1 agonist therapy directed at nigral neurons may offer a promising new avenue of neuroprotective therapy, the evidence presented here and in other reports (Heiman et al., 2014; Sodersten et al., 2014) suggests that induction of extranigral Nurr1, specifically within the striatum, is a critical trigger for the induction of LID. We hypothesized that if systemic administration of drugs that activate Nurr1 were to be employed in $\mathrm{PD}$ for neuroprotection, this could result in the exacerbation of LID, further compromising quality of life. To provide the first proof-of-principle evidence, we used the FDA-approved antimalarial drug AQ, which has been demonstrated in human neuroblastoma cells expressing Nurr1 to facilitate the transactivation of Nurr1 thru direct binding to the Nurr1 ligand binding domain and provide neuroprotection against 6-OHDA-induced nigral DA neuron loss in an in vivo rat model (Kim et al., 2015). We demonstrate here that daily AQ given in the presence of either LDOPA or the D2/3 receptor agonist ropinirole, a drug with less dyskinesia liability and is often used in early PD (Hauser et al., 2007; Bastide et al., 2015; Zesiewicz et al., 2017a) significantly exacerbates expression of AIMs in parkinsonian rats. These data suggest that systemic administration of Nurrl activating drugs with DA agonist therapy may be contraindicated in PD once DA agonist therapy is initiated.

\section{Clinical indications for Nurr1 in PD: suppressing activation of a repressed gene}

An immediate implication of our results is the potential for local striatal Nurr1 silencing in the treatment of LID as Nurr1 
activation is largely ectopic by nature in the dyskinetic striatum. Specifically, whereas other NR4A transcription factors are expressed endogenously in the striatum, in the non-dyskinetic striatum Nurr1 is undetectable (Xiao et al., 1996; Zetterstrom et al., 1996; Sodersten et al., 2014). This unique gene profile could provide a powerful therapeutic advantage since targeted inhibition of striatal Nurr1 would be anticipated to result in no adverse physiological events because its expression within the striatum is aberrant. This would contrast targeted inhibition of other striatal LID-associated genes that are known to be integral and necessary for normal neuronal function. For instance, administration of isradipine has been used to inhibit L-type $\mathrm{CaV}$ channels in preclinical models of LID. However, although such approaches have been effective, the ubiquitous expression pattern of these channels could lead to adverse off-target effects.

\section{Conclusions}

Data presented here demonstrate that Nurr1 is not only a marker of LID in preclinical models and is evident in dyskinetic human PD patients, but is directly involved in LID development, expression, and severity. We further demonstrate that ectopic Nurr1 expression is capable of facilitating aberrant or pathologic corticostriatal transmission and striatal projection neuron activity, as well as altering neuronal spine morphology in a way that mimics the disturbances in circuit neuroplasticity reported by other investigators (changes in spine morphology and synaptic plasticity resulting in persistent LTP-like changes or increases in synaptic scaling; McNeill et al., 1988; Picconi et al., 2003; Bagetta et al., 2010; Zhang et al., 2013; Fieblinger et al., 2014; Nishijima et al., 2014; Prescott et al., 2014; Spiga et al., 2014; Suarez et al., 2014, 2016; Maiti et al., 2015; Ryan et al., 2018; Villalba and Smith, 2018), and could promote LID. This characterization of Nurr1 highlights its important and potentially, causative role in LID, suggesting that Nurr1 agonists-based therapies for neuroprotection in PD may be contraindicated but that targeted silencing may be uniquely poised to provide a safe and effective anti-dyskinetic treatment option.

\section{References}

Ahlskog JE, Muenter MD (2001) Frequency of levodopa-related dyskinesias and motor fluctuations as estimated from the cumulative literature. Mov Disord 16:448-458.

Bagetta V, Ghiglieri V, Sgobio C, Calabresi P, Picconi B (2010) Synaptic dysfunction in Parkinson's disease. Biochem Soc Trans 38:493-497.

Bastide MF, Meissner WG, Picconi B, Fasano S, Fernagut PO, Feyder M, Francardo V, Alcacer C, Ding Y, Brambilla R, Fisone G, Jon Stoessl A, Bourdenx M, Engeln M, Navailles S, De Deurwaerdère P, Ko WK, Simola N, Morelli M, Groc L, et al. (2015) Pathophysiology of L-dopa-induced motor and non-motor complications in Parkinson's disease. Prog Neurobiol 132:96-168.

Beaumont V, Zhong S, Lin H, Xu W, Bradaia A, Steidl E, Gleyzes M, Wadel K, Buisson B, Padovan-Neto FE, Chakroborty S, Ward KM, Harms JF, Beltran J, Kwan M, Ghavami A, Häggkvist J, Tóth M, Halldin C, Varrone A, et al. (2016) Phosphodiesterase 10A inhibition improves cortico-basal ganglia function in Huntington's disease models. Neuron 92:1220-1237.

Benskey MJ, Manfredsson FP (2016) Intraparenchymal stereotaxic delivery of rAAV and special considerations in vector handling. Methods Mol Biol 1382:199-215.

Benskey MJ, Sandoval IM, Manfredsson FP (2016) Continuous collection of adeno-associated virus from producer cell medium significantly increases total viral yield. Hum Gene Ther Methods 27:32-45.

Benskey MJ, Sellnow RC, Sandoval IM, Sortwell CE, Lipton JW, Manfredsson FP (2018) Silencing alpha synuclein in mature nigral neurons results in rapid neuroinflammation and subsequent toxicity. Front Mol Neurosci 11:36.
Brodkin ES, Carlezon WA Jr, Haile CN, Kosten TA, Heninger GR, Nestler EJ (1998) Genetic analysis of behavioral, neuroendocrine, and biochemical parameters in inbred rodents: initial studies in Lewis and Fischer 344 rats and in A/J and C57BL/6J mice. Brain Res 805:55-68.

Bunner KD, Rebec GV (2016) Corticostriatal dysfunction in Huntington's disease: the basics. Front Hum Neurosci 10:317.

Campos-Melo D, Galleguillos D, Sánchez N, Gysling K, Andrés ME (2013) Nur transcription factors in stress and addiction. Front Mol Neurosci 6:44.

Carta M, Carlsson T, Muñoz A, Kirik D, Björklund A (2008) Involvement of the serotonin system in L-DOPA-induced dyskinesias. Parkinsonism Relat Disord 14: S154-S158.

Cepeda C, Wu N, Andre VM, Cummings DM, Levine MS (2007) The corticostriatal pathway in Huntington's disease. Prog Neurobiol 81:253-271.

Chen Y, Wang Y, Erturk A, Kallop D, Jiang Z, Weimer RM, Kaminker J, Sheng M (2014) Activity-induced Nr4al regulates spine density and distribution pattern of excitatory synapses in pyramidal neurons. Neuron 83:431-443.

Colon-Cesario WI, Martinez-Montemayor MM, Morales S, Felix J, Cruz J, Adorno M, Pereira L, Colon N, Maldonado-Vlaar CS, Pena de Ortiz S (2006) Knockdown of Nurr1 in the rat hippocampus: implications to spatial discrimination learning and memory. Learn Mem 13:734-744.

Cotzias GC, Van Woert MH, Schiffer LM (1967) Aromatic amino acids and modification of parkinsonism. N Engl J Med 276:374-379.

Day M, Wang Z, Ding J, An X, Ingham CA, Shering AF, Wokosin D, Ilijic E, Sun Z, Sampson AR, Mugnaini E, Deutch AY, Sesack SR, Arbuthnott GW, Surmeier DJ (2006) Selective elimination of glutamatergic synapses on striatopallidal neurons in Parkinson disease models. Nat Neurosci 9:251-259.

De Miranda BR, Popichak KA, Hammond SL, Jorgensen BA, Phillips AT, Safe S, Tjalkens RB (2015) The Nurr1 activator 1,1-bis(3'-indolyl)-1-(pchlorophenyl)methane blocks inflammatory gene expression in BV-2 microglial cells by inhibiting nuclear factor $\kappa$ B. Mol Pharmacol 87:10211034.

Decressac M, Volakakis N, Björklund A, Perlmann T (2013) NURR1 in Parkinson disease-from pathogenesis to therapeutic potential. Nat Rev Neurol 9:629-636.

Dekker MC, Bonifati V, van Duijn CM (2003) Parkinson's disease: piecing together a genetic jigsaw. Brain 126:1722-1733.

Dong J, Li S, Mo JL, Cai HB, Le WD (2016) Nurr1-based therapies for Parkinson's disease. CNS Neurosci Ther 22:351-359.

Fieblinger T, Cenci MA (2015) Zooming in on the small: the plasticity of striatal dendritic spines in L-DOPA-induced dyskinesia. Mov Disord 30:484-493.

Fieblinger T, Graves SM, Sebel LE, Alcacer C, Plotkin JL, Gertler TS, Chan CS, Heiman M, Greengard P, Cenci MA, Surmeier DJ (2014) Cell typespecific plasticity of striatal projection neurons in parkinsonism and LDOPA-induced dyskinesia. Nat Commun 5:5316.

Fole A, Miguens M, Morales L, Gonzalez-Martin C, Ambrosio E, Del Olmo N (2017) Lewis and Fischer 344 rats as a model for genetic differences in spatial learning and memory: cocaine effects. Prog Neuropsychopharmacol Biol Psychiatry 76:49-57.

Fox SH, Katzenschlager R, Lim SY, Ravina B, Seppi K, Coelho M, Poewe W, Rascol O, Goetz CG, Sampaio C (2011) The movement disorder society evidence-based medicine review update: treatments for the motor symptoms of Parkinson's disease. Mov Disord 26:S2-S41.

Gertler TS, Chan CS, Surmeier DJ (2008) Dichotomous anatomical properties of adult striatal medium spiny neurons. J Neurosci 28:10814-10824.

Giguere V (1999) Orphan nuclear receptors: from gene to function. Endocr Rev 20:689-725.

Groves PM (1983) A theory of the functional organization of the neostriatum and the neostriatal control of voluntary movement. Brain Res 5:109-132.

Hauser RA, Rascol O, Korczyn AD, Jon Stoessl A, Watts RL, Poewe W, De Deyn PP, Lang AE (2007) Ten-year follow-up of Parkinson's disease patients randomized to initial therapy with ropinirole or levodopa. Mov Disord 22:2409-2417.

Hawk JD, Bookout AL, Poplawski SG, Bridi M, Rao AJ, Sulewski ME, Kroener BT, Manglesdorf DJ, Abel T (2012) NR4A nuclear receptors support memory enhancement by histone deacetylase inhibitors. J Clin Invest 122:3593-3602.

Heiman M, Heilbut A, Francardo V, Kulicke R, Fenster RJ, Kolaczyk ED, Mesirov JP, Surmeier DJ, Cenci MA, Greengard P (2014) Molecular 
adaptations of striatal spiny projection neurons during levodopa-induced dyskinesia. Proc Natl Acad Sci U S A 111:4578-4583.

Ingham CA, Hood SH, Arbuthnott GW (1989) Spine density on neostriatal neurones changes with 6-hydroxydopamine lesions and with age. Brain Res 503:334-338.

Jiang C, Wan X, He Y, Pan T, Jankovic J, Le W (2005) Age-dependent dopaminergic dysfunction in Nurr1 knockout mice. Exp Neurol 191:154-162.

Johnson MM, Michelhaugh SK, Bouhamdan M, Schmidt CJ, Bannon MJ (2011) The transcription factor NURR1 exerts concentration-dependent effects on target genes mediating distinct biological processes. Front. Neurosci 5:135.

Kadkhodaei B, Ito T, Joodmardi E, Mattsson B, Rouillard C, Carta M, Muramatsu S, Sumi-Ichinose C, Nomura T, Metzger D, Chambon P, Lindqvist E, Larsson NG, Olson L, Bjorklund A, Ichinose H, Perlmann T (2009) Nurrl is required for maintenance of maturing and adult midbrain dopamine neurons. J Neurosci 29:15923-15932.

Kim CH, Han BS, Moon J, Kim DJ, Shin J, Rajan S, Nguyen QT, Sohn M, Kim WG, Han M, Jeong I, Kim KS, Lee EH, Tu Y, Naffin-Olivos JL, Park CH, Ringe D, Yoon HS, Petsko GA, Kim KS (2015) Nuclear receptor Nurr1 agonists enhance its dual functions and improve behavioral deficits in an animal model of Parkinson's disease. Proc Natl Acad Sci U S A 112:8756-8761.

Konradi C, Westin JE, Carta M, Eaton ME, Kuter K, Dekundy A, Lundblad M, Cenci MA (2004) Transcriptome analysis in a rat model of L-DOPAinduced dyskinesia. Neurobiol Dis 17:219-236.

Lane EL, Dunnett SB (2010) Pre-treatment with dopamine agonists influence L-dopa mediated rotations without affecting abnormal involuntary movements in the 6-OHDA lesioned rat. Behav Brain Res 213:66-72.

Le WD, Xu P, Jankovic J, Jiang H, Appel SH, Smith RG, Vassilatis DK (2003) Mutations in NR4A2 associated with familial Parkinson disease. Nat Genet 33:85-89.

Levine ND, Rademacher DJ, Collier TJ, O’Malley JA, Kells AP, San Sebastian W, Bankiewicz KS, Steece-Collier K (2013) Advances in thin tissue GolgiCox impregnation: fast, reliable methods for multi-assay analyses in rodent and non-human primate brain. J Neurosci Methods 213:214-227.

Lu XY, Ghasemzadeh MB, Kalivas PW (1997) Expression of D1 receptor, D2 receptor, substance $\mathrm{P}$ and enkephalin messenger RNAs in the neurons projecting from the nucleus accumbens. Neuroscience 82:767-780.

Lundblad M, Andersson M, Winkler C, Kirik D, Wierup N, Cenci MA (2002) Pharmacological validation of behavioural measures of akinesia and dyskinesia in a rat model of Parkinson's disease. Eur J Neurosci 15:120-132.

Mahmoudi S, Samadi P, Gilbert F, Ouattara B, Morissette M, Grégoire L, Rouillard C, Di Paolo T, Lévesque D (2009) Nur77 mRNA levels and LDopa-induced dyskinesias in MPTP monkeys treated with docosahexaenoic acid. Neurobiol Dis 36:213-222.

Maira M, Martens C, Philips A, Drouin J (1999) Heterodimerization between members of the Nur subfamily of orphan nuclear receptors as a novel mechanism for gene activation. Mol Cell Biol 19:7549-7557.

Maiti P, Manna J, Ilavazhagan G, Rossignol J, Dunbar GL (2015) Molecular regulation of dendritic spine dynamics and their potential impact on synaptic plasticity and neurological diseases. Neurosci Biobehav Rev 59:208237.

Manson A, Stirpe P, Schrag A (2012) Levodopa-induced-dyskinesias clinical features, incidence, risk factors, management and impact on quality of life. J Parkinsons Dis 2:189-198.

Maries E, Kordower JH, Chu Y, Collier TJ, Sortwell CE, Olaru E, Shannon K, Steece-Collier K (2006) Focal not widespread grafts induce novel dyskinetic behavior in parkinsonian rats. Neurobiol Dis 21:165-180.

McNeill TH, Brown SA, Rafols JA, Shoulson I (1988) Atrophy of medium spiny I striatal dendrites in advanced Parkinson's disease. Brain Res 455:148-152.

Miguens M, Coria SM, Higuera-Matas A, Fole A, Ambrosio E, Del Olmo N (2011) Depotentiation of hippocampal long-term potentiation depends on genetic background and is modulated by cocaine self-administration. Neuroscience 187:36-42.

Millan MJ, Di Cara B, Hill M, Jackson M, Joyce JN, Brotchie J, McGuire S, Crossman A, Smith L, Jenner P, Gobert A, Peglion JL, Brocco M (2004) S32504, a novel naphtoxazine agonist at dopamine D3/D2 receptors: II. Actions in rodent, primate, and cellular models of antiparkinsonian activity in comparison to ropinirole. J Pharmacol Exp Ther 309:921-935.
Nishijima H, Suzuki S, Kon T, Funamizu Y, Ueno T, Haga R, Suzuki C, Arai A, Kimura T, Suzuki C, Meguro R, Miki Y, Yamada J, Migita K, Ichinohe N, Ueno S, Baba M, Tomiyama M (2014) Morphologic changes of dendritic spines of striatal neurons in the levodopa-induced dyskinesia model. Mov Disord 29:336-343.

Padovan-Neto FE, Sammut S, Chakroborty S, Dec AM, Threlfell S, Campbell PW, Mudrakola V, Harms JF, Schmidt CJ, West AR (2015) Facilitation of corticostriatal transmission following pharmacological inhibition of striatal phosphodiesterase 10A: role of nitric oxide-soluble guanylyl cyclase-cGMP signaling pathways. J Neurosci 35:5781-5791.

Papathanou M, Rose S, McCreary A, Jenner P (2011) Induction and expression of abnormal involuntary movements is related to the duration of dopaminergic stimulation in 6-OHDA-lesioned rats. Eur J Neurosci 33:2247-2254.

Pena de Ortiz S, Maldonado-Vlaar CS, Carrasquillo Y (2000) Hippocampal expression of the orphan nuclear receptor gene hzf-3/nurrl during spatial discrimination learning. Neurobiol Learn Mem 74:161-178.

Picconi B, Centonze D, Hakansson K, Bernardi G, Greengard P, Fisone G, Cenci MA, Calabresi P (2003) Loss of bidirectional striatal synaptic plasticity in L-DOPA-induced dyskinesia. Nat Neurosci 6:501-506.

Pollack AE, Yates TM (1999) Prior D1 dopamine receptor stimulation is required to prime D2-mediated striatal Fos expression in 6-hydroxydopamine-lesioned rats. Neuroscience 94:505-514.

Pollack AE, Thomas LI (2010) D1 priming enhances both D1- and D2-mediated rotational behavior and striatal Fos expression in 6-hydroxydopamine lesioned rats. Pharmacol Biochem Behav 94:346-351.

Prescott IA, Liu LD, Dostrovsky JO, Hodaie M, Lozano AM, Hutchison WD (2014) Lack of depotentiation at basal ganglia output neurons in PD patients with levodopa-induced dyskinesia. Neurobiol Dis 71:24-33.

Rouillard C, Baillargeon J, Paquet B, St-Hilaire M, Maheux J, Lévesque C, Darlix N, Majeur S, Lévesque D (2018) Genetic disruption of the nuclear receptor Nur77 (Nr4a1) in rat reduces dopamine cell loss and L-DOPAinduced dyskinesia in experimental Parkinson's disease. Exp Neurol 304:143-153.

Ryan MB, Bair-Marshall C, Nelson AB (2018) Aberrant striatal activity in parkinsonism and levodopa-induced dyskinesia. Cell Rep 23:3438-3446. e5.

Sammut S, Threlfell S, West AR (2010) Nitric oxide-soluble guanylyl cyclase signaling regulates corticostriatal transmission and short-term synaptic plasticity of striatal projection neurons recorded in vivo. Neuropharmacology 58:624-631.

Sandoval IM, Kuhn NM, Manfredsson FP (2019) Multimodal production of adeno-associated virus. Methods Mol Biol 1937:101-124.

Schwarting RK, Huston JP (1996) The unilateral 6-hydroxydopamine lesion model in behavioral brain research. Analysis of functional deficits, recovery and treatments. Prog Neurobiol 50:275-331.

Sellnow RC, Steece-Collier K, Kanaan NM, Sortwell CE, Collier TJ, ColeStrauss A, Lipton JW, Manfredsson FP (2015) rAAV-mediated regulation of striatal nurr1 expression alters development and severity of levodopainduced dyskinesias in the 6-OHDA rat model of Parkinson's disease. Mol Ther 23:S282-S283.

Sellnow RC, Newman JH, Chambers N, West AR, Steece-Collier K, Sandoval IM, Benskey MJ, Bishop C, Manfredsson FP (2019) Regulation of dopamine neurotransmission from serotonergic neurons by ectopic expression of the dopamine D2 autoreceptor blocks levodopa-induced dyskinesia. Acta Neuropathol Commun 7:8.

Sepers MD, Raymond LA (2014) Mechanisms of synaptic dysfunction and excitotoxicity in Huntington's disease. Drug Discov Today 19:990-996.

Simuni T (2018) Longitudinal change of clinical and biological measures in early Parkinson's disease: Parkinson's progression markers initiative cohort. Mov Disord 33:771-782.

Smith GA, Rocha EM, Rooney T, Barneoud P, McLean JR, Beagan J, Osborn T, Coimbra M, Luo Y, Hallett PJ, Isacson O (2015) A Nurrl agonist causes neuroprotection in a Parkinson's disease lesion model primed with the toll-like receptor 3 dsRNA inflammatory stimulant poly(I: c). PLoS One 10:e121072.

Sodersten E, Feyder M, Lerdrup M, Gomes AL, Kryh H, Spigolon G, Caboche J, Fisone G, Hansen K (2014) Dopamine signaling leads to loss of Polycomb repression and aberrant gene activation in experimental parkinsonism. PLoS Genet 10:e1004574.

Soderstrom KE, O'Malley JA, Levine ND, Sortwell CE, Collier TJ, SteeceCollier K (2010) Impact of dendritic spine preservation in medium spiny 
neurons on dopamine graft efficacy and the expression of dyskinesias in parkinsonian rats. Eur J Neurosci 31:478-490.

Spiga S, Mulas G, Piras F, Diana M (2014) The "addicted" spine. Front Neuroanat 8:110.

St-Hilaire M, Landry E, Levesque D, Rouillard C (2003) Denervation and repeated L-DOPA induce a coordinate expression of the transcription factor NGFI-B in striatal projection pathways in hemi-parkinsonian rats. Neurobiol Dis 14:98-109.

Stanika R, Campiglio M, Pinggera A, Lee A, Striessnig J, Flucher BE, Obermair GJ (2016) Splice variants of the CaV1.3 L-type calcium channel regulate dendritic spine morphology. Sci Rep 6:34528.

Steece-Collier K, Collier TJ, Danielson PD, Kurlan R, Yurek DM, Sladek JR Jr (2003) Embryonic mesencephalic grafts increase levodopa-induced forelimb hyperkinesia in parkinsonian rats. Mov Disord 18:1442-1454.

Steece-Collier K, Stancati JA, Collier NJ, Sandoval IM, Mercado NM, Sortwell CE, Collier TJ, Manfredsson FP (2019) Genetic silencing of striatal CaV1.3 prevents and ameliorates levodopa dyskinesia. Mov Disord 34:697-707.

Steece-Collier K, Collier JC, Lipton JW, Stancati JA, Winn ME, Cole-Strauss A, Sellnow R, Conti MM, Mercado NM, Nillni EA, Sortwell CE, Manfredsson FP, Bishop C (2020) Striatal Nurr1, but not FosB expression links a levodopa-induced dyskinesia phenotype to genotype in Fisher 344 vs. Lewis hemiparkinsonian rats. Exp Neurol, in press.

Suarez LM, Solis O, Aguado C, Lujan R, Moratalla R (2016) L-DOPA oppositely regulates synaptic strength and spine morphology in D1 and D2 striatal projection neurons in dyskinesia. Cereb Cortex 26:4253-4264.

Suarez LM, Solis O, Carames JM, Taravini IR, Solis JM, Murer MG, Moratalla R (2014) L-DOPA treatment selectively restores spine density in dopamine receptor D2-expressing projection neurons in dyskinetic mice. Biol Psychiatry 75:711-722.

Threlfell S, Sammut S, Menniti FS, Schmidt CJ, West AR (2009) Inhibition of phosphodiesterase $10 \mathrm{~A}$ increases the responsiveness of striatal projection neurons to cortical stimulation. J Pharmacol Exp Ther 328:785-795.

Tokuoka H, Hatanaka T, Metzger D, Ichinose H (2014) Nurrl expression is regulated by voltage-dependent calcium channels and calcineurin in cultured hippocampal neurons. Neurosci Lett 559:50-55.
Valenza M, Picetti R, Yuferov V, Butelman ER, Kreek MJ (2016) Strain and cocaine-induced differential opioid gene expression may predispose Lewis but not Fischer rats to escalate cocaine self-administration. Neuropharmacology 105:639-650.

Villalba RM, Smith Y (2018) Loss and remodeling of striatal dendritic spines in Parkinson's disease: from homeostasis to maladaptive plasticity? J Neural Transm 125:431-447.

Volakakis N, Tiklova K, Decressac M, Papathanou M, Mattsson B, Gillberg L, Nobre A, Bjorklund A, Perlmann T (2015) Nurrl and retinoid X receptor ligands stimulate ret signaling in dopamine neurons and can alleviate alpha-synuclein disrupted gene expression. J Neurosci 35:1437014385 .

Xiao Q, Castillo SO, Nikodem VM (1996) Distribution of messenger RNAs for the orphan nuclear receptors Nurrl and Nur77 (NGFI-B) in adult rat brain using in situ hybridization. Neuroscience 75:221-230.

Yamada H, Kuroki T, Nakahara T, Hashimoto K, Tsutsumi T, Hirano M, Maeda H (2007) The dopamine D1 receptor agonist, but not the D2 receptor agonist, induces gene expression of Homer 1a in rat striatum and nucleus accumbens. Brain Res 1131:88-96.

Zesiewicz TA, Chriscoe S, Jimenez T, Upward J, VanMeter S (2017a) A fixed-dose, dose-response study of ropinirole prolonged release in early stage Parkinson's disease. Neurodegener Dis Manag 7:49-59.

Zesiewicz TA, Chriscoe S, Jimenez T, Upward J, Davy M, VanMeter S (2017b) A randomized, fixed-dose, dose-response study of ropinirole prolonged release in advanced Parkinson's disease. Neurodegener Dis Manag 7:61-72.

Zetterstrom RH, Williams R, Perlmann T, Olson L (1996) Cellular expression of the immediate early transcription factors Nurrl and NGFI-B suggests a gene regulatory role in several brain regions including the nigrostriatal dopamine system. Brain Res Mol Brain Res 41:111-120.

Zetterstrom RH, Solomin L, Jansson L, Hoffer BJ, Olson L, Perlmann T (1997) Dopamine neuron agenesis in Nurrl-deficient mice. Science 276:248-250.

Zhang Y, Meredith GE, Mendoza-Elias N, Rademacher DJ, Tseng KY, Steece-Collier K (2013) Aberrant restoration of spines and their synapses in L-DOPA-induced dyskinesia: involvement of corticostriatal but not thalamostriatal synapses. J Neurosci 33:11655-11667. 